\title{
Velocity Structure of the Interstellar Medium as Seen by the Spectral Correlation Function
}

\section{Citation}

Ballesteros\#Paredes, Javier, Enrique Vazquez\#Semadeni, and Alyssa A. Goodman. 2002. "Velocity Structure of the Interstellar Medium as Seen by the Spectral Correlation Function." The Astrophysical Journal 571 (1): 334-55. https://doi.org/10.1086/339875.

\section{Permanent link}

http://nrs.harvard.edu/urn-3:HUL.InstRepos:41397409

\section{Terms of Use}

This article was downloaded from Harvard University's DASH repository, and is made available under the terms and conditions applicable to Other Posted Material, as set forth at http:// nrs.harvard.edu/urn-3:HUL.InstRepos:dash.current.terms-of-use\#LAA

\section{Share Your Story}

The Harvard community has made this article openly available.

Please share how this access benefits you. Submit a story.

Accessibility 


\title{
VELOCITY STRUCTURE OF THE INTERSTELLAR MEDIUM AS SEEN BY THE SPECTRAL CORRELATION FUNCTION
}

\author{
Javier Ballesteros-Paredes, ${ }^{1,2,3}$ Enrique VÁzquez-Semadeni, ${ }^{2}$ and Alyssa A. Goodman ${ }^{3}$ \\ Received 2001 May 28; accepted 2002 January 28
}

\begin{abstract}
We use the statistical tool known as the "spectral correlation function" (SCF) to intercompare simulations and observations of the atomic interstellar medium (ISM). The simulations considered, which mimic three distinct sets of physical conditions, are each calculated for a $300 \mathrm{pc}^{3}$ box centered at the Galactic plane. The "ISM" run is intended to represent a mixture of cool and warm atomic gas and includes self-gravity and magnetic fields in the calculations. The "ISM-IT" run is more representative of molecular clouds, in which the gas is presumed isothermal. The third run "IT" is for purely isothermal gas, with zero magnetic field and no self-gravity. Forcing in the three cases is accomplished by including simulated effects of stellar heating (ISM), stellar winds (ISM-IT), or random compressible fluctuations (IT). For each simulation, H i spectral line maps are simulated, and it is these maps that are intercompared, both with each other and with observations, using the SCF. For runs where the separation of velocity features is much greater than the "thermal" width of a line, density-weighted velocity histograms are decent estimates of $\mathrm{H}$ i spectra. When thermal broadening is large in comparison with fine-scale turbulent velocity structure, this broadening masks subthermal velocity substructure in observed spectra. So, simulated spectra for runs in which thermal broadening is important must be calculated by convolving density-weighted histograms with Gaussians whose width represents the thermal broadening. The $\mathrm{H}$ i observations we use for comparison are of the north celestial pole (NCP) loop, a region chosen to minimize line-of-sight confusion on scales greater than $100 \mathrm{pc}$. None of the simulations match the NCP loop data very well, for a variety of reasons described in the paper. Most of the reasons for simulation/observation discrepancy are predictable and understandable, but one is particularly interesting: the most realistic sets of line profiles and SCF statistics come from artificially expanding the velocity axis of the ISM run by a factor of 6 . Without rescaling, the low-velocity dispersion associated with much of the gas in the ISM run causes almost all of the spectra to appear as virtually identical Gaussians whose width is determined solely by temperature - all velocity structure is smeared out by thermal broadening. However, if the velocity axis is expanded by a factor of 6,the SCF distributions of the ISM run and the NCP loop match up fairly well. This means that the ratio of thermal to turbulent pressure in the ISM simulation is much too large as it stands, and that the simulation is deficient in turbulent energy. This is a consequence of the ISM run not including the effects of supernovae. This paper concludes that the SCF is a useful tool for understanding and fine-tuning simulations of interstellar gas, and in particular that realistic simulations of the atomic ISM need to include the effects of energetic stellar winds (e.g., supernovae) in order for the ratio of thermal-to-turbulent pressure to give spectra representative of the observed ISM in our Galaxy.
\end{abstract}

Subject headings: ISM: clouds - ISM: kinematics and dynamics — methods: data analysis methods: statistical — turbulence

\section{INTRODUCTION}

In the past few decades, the turbulent character of the velocity field in the interstellar medium has been increasingly recognized (Zuckerman \& Evans 1974; Dickman 1985; Scalo 1987; for recent works, see the volume by Franco \& Carramiñana 1999 and the review by VázquezSemadeni et al. 2000b). Due to the highly fluctuating character and sensitivity to initial conditions (initial conditions arbitrarily close to each other separate exponentially in time - see, e.g., Lesieur 1990) of the turbulent motions, statistical tools become important in trying to characterize and understand the nature of interstellar turbulence. These tools become even more important with the continuous growth of

\footnotetext{
${ }^{1}$ Astrophysics Department, American Museum of Natural History, Central Park West at 79th Street, New York, NY 10024; javierbp@amnh.org.

2 Instituto de Astronomía, Universidad Nacional Autónoma de México, Apartado Postal 70-264, 04510 México D.F., Mexico.

${ }^{3}$ Harvard-Smithsonian Center for Astrophysics, 60 Garden Street, MS-42, Cambridge, MA 02138.
}

both observed and theoretical data cubes, as it becomes necessary to compress such large data sets into a more manageable parameter space. On the observational side, studies of the centroid velocity probability distribution function ( $v$-PDF; Miesch \& Scalo 1995; Lis et al. 1996; Miesch, Scalo, $\&$ Bally 1999) have compared the shape of the $v$-PDF for various observed regions and numerical simulations of incompressible or weakly compressible turbulence; through the density and velocity autocorrelation functions, Kleiner \& Dickman (1984) have tried to infer the correlation length scale in the Taurus molecular cloud; using structure tree statistics, Houlahan \& Scalo (1992) tried to discriminate between hierarchically nested and random collections of clouds; through principal component analysis and autocorrelation functions, Heyer \& Schloerb (1997) and Brunt \& Heyer (2001) obtain the velocity dispersion-size relationship for both observations and pseudosimulations, in a manner independent of how clouds are defined; applying $\Delta$ variance analysis, Stutzki et al. (1998) and Bensch, Stutski, \& Osenkopf (2001) have measured the fractal dimension of the projected intensity toward the Polaris Flare. Finally, 
Lazarian, \& Pogosyan (2000) have given a method for recovering the power spectra of both the three-dimensional density and of the line-of-sight velocity from the emissivity power spectrum in velocity channels of spectroscopic data.

On the numerical side, recent simulations of interstellar turbulence have allowed statistical studies of the simulated ISM and clouds. In fact, again because of the sensitivity to initial conditions, modeling of individual turbulent objects is not feasible, and statistical modeling is again the appropriate way to proceed. It, thus, becomes appropriate to compare numerical simulations and observations using statistical methods. For example, Falgarone et al. (1994) studied optically thin line profiles in $512^{3}$ simulations, concluding that the shapes of the spectra, their moments, and their spatial variation exhibit similarities with observational line profiles of non-star-forming regions; VázquezSemadeni, Ballesteros-Paredes, \& Rodríguez (1997) searched for Larson-type relationships in numerical simulations, finding, in particular, that the density-size relationship is not satisfied, but instead there exists a whole family of low column density clouds that may, however, be missed by observational surveys limited by integration time (see also Scalo 1990); Padoan \& Nordlund (1999) compare suband super-Alfvénic simulations with observations, concluding that the first case may be in conflict with observations; Pichardo et al. (2000) studied the projection of numerical MHD simulations, finding that the morphology of the channel maps resembles maps of the line of sight (LOS) of the velocity more closely than maps of the density field; Mac Low \& Ossenkopf (1999) have recently used the $\Delta$-variance to find that the models with high Mach numbers (more than $M \sim 4$ ) are in better agreement with observations; Lazarian et al. (2001) have shown that the theoretical predictions of Lazarian \& Pogosyan (2000) are verified for simulated spectroscopic data from numerical simulations of ISM turbulence.

In particular, Rosolowsky et al. (1999, hereafter RGWW) introduced a new method, called the "spectral correlation function" (SCF), which estimates the spatially averaged ${ }^{4}$

\footnotetext{
${ }^{4}$ In this paper the word "spatial" refers to the plane of the sky, unless otherwise stated.
}

correlation of spectra inside a box of size $s$ in spectroscopic data cubes. These authors used the SCF to measure the correlation between spectra in neighboring positions, as an indicator of the degree of "smoothness" of both observational and numerical data cubes. With this method, they were able to distinguish between, for example, simulations with and without self-gravity, by comparing the variation of the SCF upon randomization of the positions of the spectra on the plane of the sky (POS).

In the present work, we extend the work of RGWW, further exploring the capabilities of the SCF to discriminate among numerical simulations of various turbulent regimes (isothermal with large- and small-scale forcing and ISMlike), and to quantitatively compare them to spectroscopic observational data of diffuse $\mathrm{H}$ I gas. In order to do this, we construct spectral maps from the numerical data cubes assuming optically thin lines, with and without thermal broadening of lines. For nonisothermal gas, the velocity structure of the colder gas may be "hidden" by the thermal broadening of the warmer gas.

The plan of the paper is as follows: in $\S 2$ we describe both the observed H I data (Hartmann \& Burton 1997 ) and the simulated data cubes used here and explain the construction of the spectral maps for the simulations. In $\S 3$ we briefly review the SCF. In $\S 4$ we apply the SCF to the data sets, discussing the information that can be extracted from this procedure toward distinguishing between the various simulations and quantitatively estimating their similarity with observational data. In $\S 5$ we discuss the nature of the spatial structure of the SCF and discuss how the SCF can be used to guide theoreticians to match their simulations with observational data. Finally, in $\S 6$ we draw the main conclusions.

\section{DATA}

\subsection{Numerical Simulations}

We consider three numerical simulations in three dimensions. The first one, called simply ISM, represents the ISM in a box of $300 \mathrm{pc}$ on a side, at a resolution of 100 points per dimension, centered on the Galactic midplane at the solar

TABLE 1

Summary of Simulation Runs

\begin{tabular}{|c|c|c|c|}
\hline Run Name & Synthetic Line Spectra Generated & Velocity Channels & Comments \\
\hline \multirow{3}{*}{ 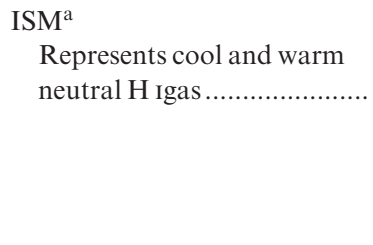 } & $\begin{array}{l}\text { NTB: Density-weighted velocity } \\
\text { histograms }\end{array}$ & 16 and 64 & Unrealistic, spiky spectra; these structures are unobservable \\
\hline & $\begin{array}{l}\text { TB: Each cell broadened by } \\
\text { temperature at that position }\end{array}$ & 16 & Spectra too smooth and uniform across the map \\
\hline & Velocity adjusted $(\times 6)$ & 16 & $\begin{array}{l}\text { More realistic spectra and SCF; shows that ISM run is less } \\
\text { turbulent than } \mathrm{H} \text { I data }\end{array}$ \\
\hline $\begin{array}{l}\text { ISM-IT }^{\mathrm{b}} \\
\text { Isothermal, but with ISM } \\
\text { ingredients; represents } \\
\text { molecular clouds ....................... }\end{array}$ & NTB & 16 & $\begin{array}{l}\text { Nonrealistic spectra; still "wormy" appearance of the } \\
\text { SCF maps }\end{array}$ \\
\hline 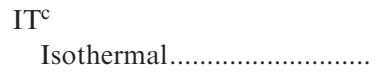 & NTB & 16 & $\begin{array}{l}\text { Nonrealistic spectra; roundish "worms" in the SCF } \\
\text { caused by the forcing }\end{array}$ \\
\hline
\end{tabular}

a Physical conditions: $300 \mathrm{pc}^{3}$ box; Galactic midplane; conditions at $R \odot$; self-gravity is on; magnetic fields are on; Jeans length $\sim 200 \mathrm{pc}$; forcing from stellar heating; density threshold for $\mathrm{SF}$ is $\rho_{\mathrm{th}}=8 \mathrm{~cm}^{-3}$.

b Physical conditions: Same as ISM but isothermal; Jeans length (isothermal); 1.1 box size; forcing from stellar "winds "; lower density threshold for SF is $\rho_{\text {th }}=4 \mathrm{~cm}^{-3}$

c Physical conditions: Pure HD, no self-gravity; isothermal; forcing is random, compressible. 

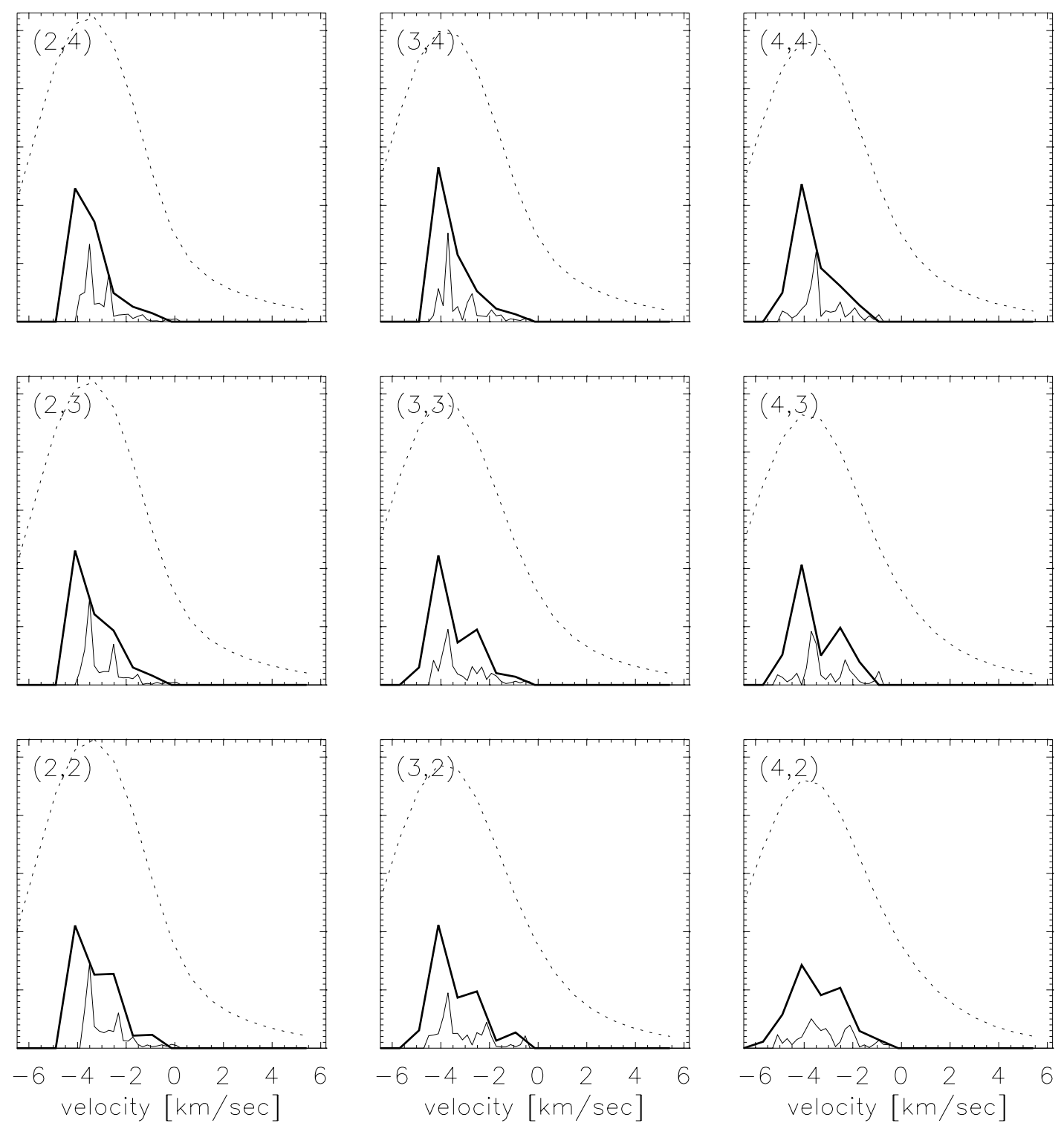

FIG. 1.-Nine line profiles for the ISM run centered at position $(3,3)$ on the simulation's POS (the $[x, y]$-plane). The thick solid lines represent the NTB line spectra at a resolution of 16 channels. The thin solid lines represent the NTB spectra with 64 velocity channels, and the dotted lines represent the TB spectra at 16 velocity channels. Note that in the latter all the spectral features are hidden by the thermal broadening, and that the high-resolution spectra are poorly sampled, as evidenced by their spiky nature.

Galactocentric distance. Full equations, details, and a complete description of the model can be found in Passot, Vázquez-Semadeni, \& Pouquet (1995, hereafter PVP) and Pichardo et al. (2000). Here we just mention that the code solves the full self-gravitating MHD equations, including that of the internal energy conservation with additional model terms representing the background plus local stellar heating and the radiative cooling. The local heating mimics stellar ionization heating and is turned on ("an O star is formed") when the local density reaches a threshold value of 10 times the mean density, producing local bubbles of warm gas that expand and feed the global turbulence. "Stars" remain on for 2 Myr. No supernova-like energy injection is included because of numerical limitations. The cooling is parameterized as a piecewise power-law function as fitted to the cooling functions of Dalgarno \& McCray (1972) and of Raymond, Cox, \& Smith (1976) by Rosen, Bregman, \& Norman (1993) and Rosen \& Bregman (1995; see PVP and Pichardo et al. 2001). The "isothermal" Jeans length is twice the integration box size, although the presence of cooling gives an effective Jeans length of roughly $\frac{2}{3}$ of the box size (Ballesteros-Paredes, Vázquez-Semadeni, \& Scalo 1999). The mean density is $3.3 \mathrm{~cm}^{-3}$ and the velocity, temperature, and magnetic field units are, respectively, 6.4 $\mathrm{km} \mathrm{s}^{-1}, 3000 \mathrm{~K}$, and $5 \mu \mathrm{G}$.

Two limitations of the ISM simulation should be mentioned. First of all, the lack of supernova energy input implies that the simulations are somewhat less energetic than the actual ISM. This fact will be quantified in $\S 5.2$. On the other hand, the heating and cooling functions used do not produce any thermally unstable temperature ranges, although we do not consider this to be a serious problem since recent discussions by Vázquez-Semadeni et al. (2000a) and Gazol et al. (2001) suggest that the structural properties of the ISM are more a consequence of the dynamic processes than of the thermal ones. On the 


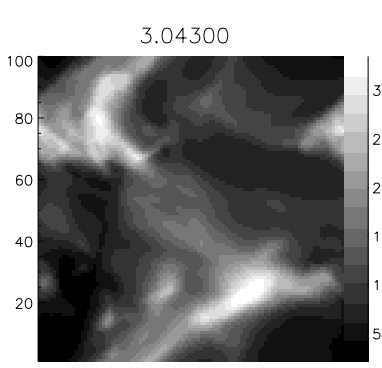

$-0.133000$

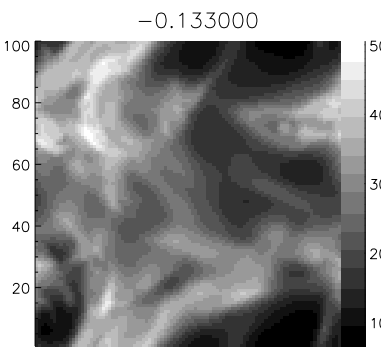

$-3.30900$

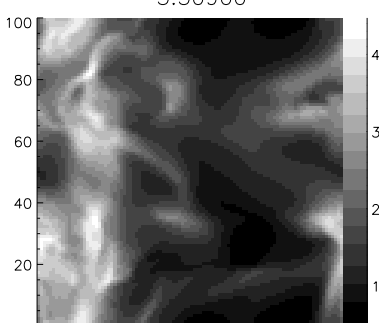

$-6.48500$

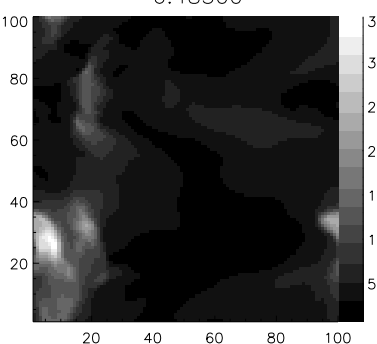

3.83700

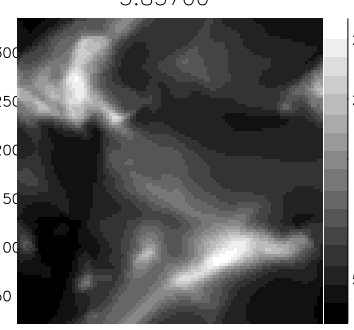

0.661000

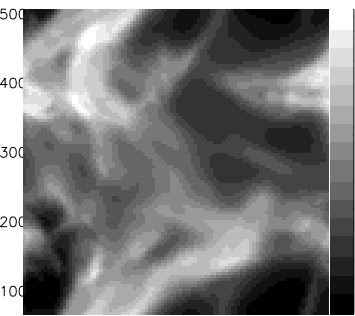

$-2.51500$

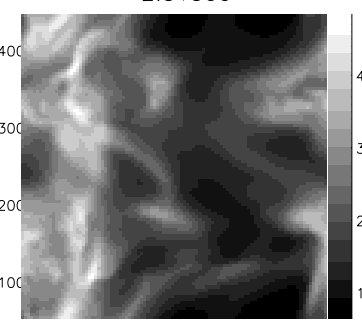

$-5.69100$

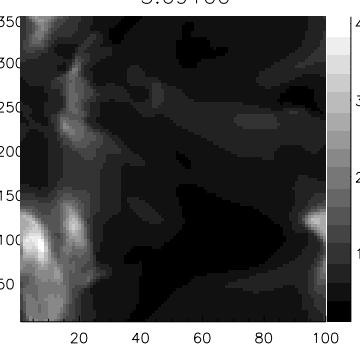

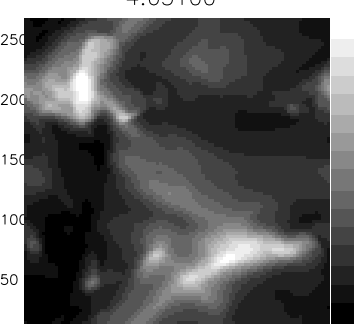

1.45500

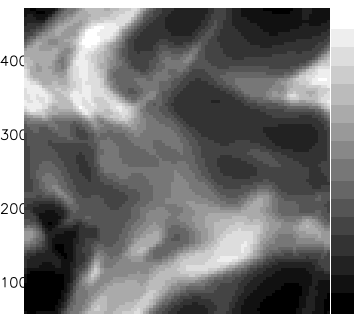

$-1.72100$

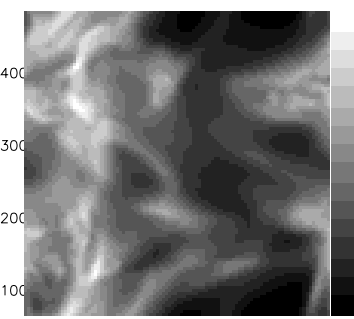

$-4.89700$

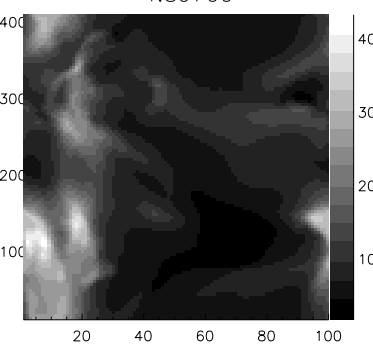

5.42500

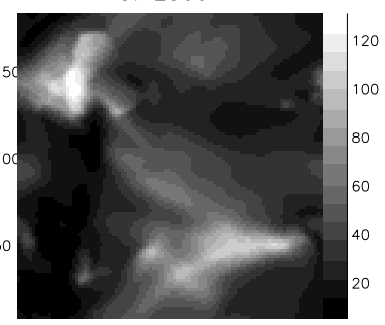

2.24900

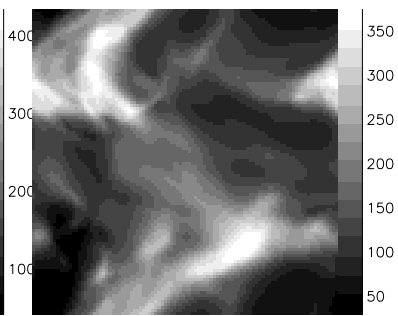

$-0.927000$

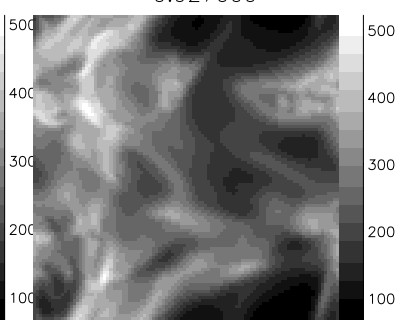

$-4.10300$

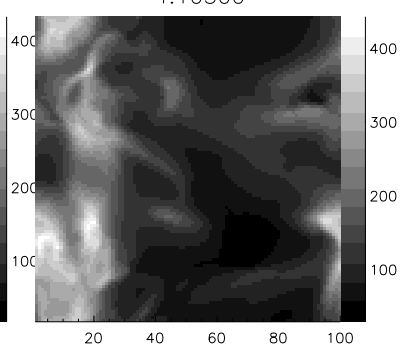

FIG. $2 a$

FIG. 2.-Velocity channel maps for the four different "observations" of the ISM run: $(a) 16$ velocity channels, TB; $(b) 16$ velocity channels, NTB; $(c) 64$ velocity channels, TB; $(d) 64$ velocity channels, NTB. The NTB maps show veil-like structures with sharp edges. The TB maps are much smoother.

other hand, a true shortcoming is the absence of hot ( $\left.T \sim 10^{6} \mathrm{~K}\right)$ gas, although recent work by various groups (e.g., Gazol-Patiño \& Passot 1999; de Avillez 2000) suggests that the filling factor of this gas at the midplane is not larger than $\sim 20 \%$. In this case, the error committed by not including it is probably minor since the main effect of this omission will be that in actual observations a fraction of the LOS will contain "holes" in the cold/ warm gas distribution, filled with hot ionized gas, which will be absent in our simulation data. Given the low resolution of our simulations, this may actually constitute an advantage since better sampling of the gas contributing to the $\mathrm{H}$ I line spectra along each LOS will be accomplished.

The second simulation, called ISM-IT, is similar to the ISM run, except that an isothermal regime is considered, with no cooling and heating terms, the isothermal Jeans length is 1.1 times the box size, and a lower density thresh- old is used for initiating star formation so that the star formation rate is similar to that of the ISM run in spite of the weaker gravity. In this run, which may be thought to represent gas within a molecular cloud at $T \sim 10 \mathrm{~K}$, the stellar energy input proceeds via winds (locally divergent source terms in the momentum equation-see Vázquez-Semadeni, Passot, \& Pouquet 1996; Avila-Reese \& Vázquez-Semadeni 2001), and the internal energy evolution equation is bypassed altogether, but self-gravity and the magnetic field are still included. Finally, the third run, also at a resolution of $100^{3}$ and called IT, is a purely hydrodynamic isothermal run without self-gravity and with random compressible forcing applied at scales of $\frac{1}{4}$ the simulation box size. As a summary, we present in Table 1 the main features of each one of the runs.

All three runs are started with Gaussian fluctuations with random phases, uncorrelated among all variables. Note that for the IT run, the forcing is active from the beginning of the 

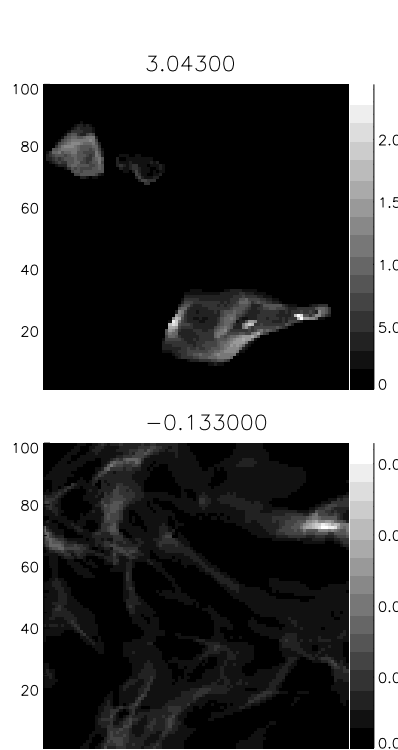

$-3.30900$

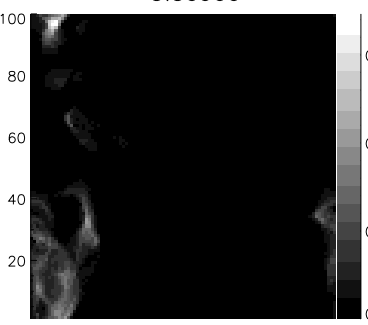

$-6.48500$

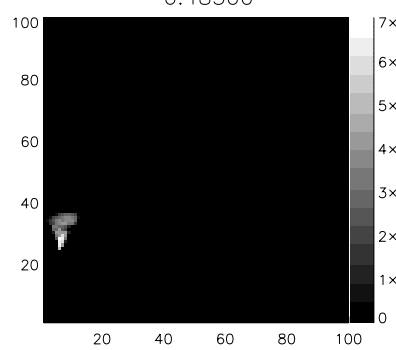

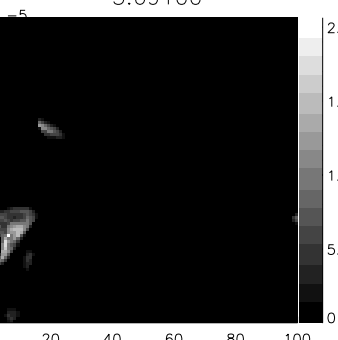

ISM100 16ch $t=3.3$ NTB

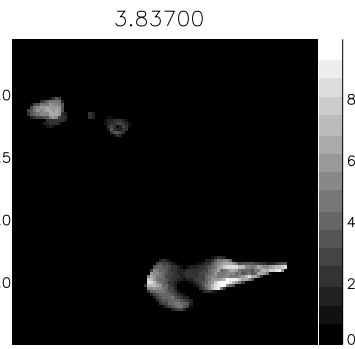

0.661000

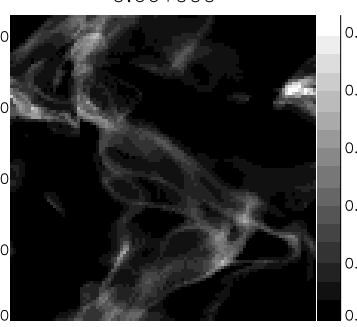

$-2.51500$

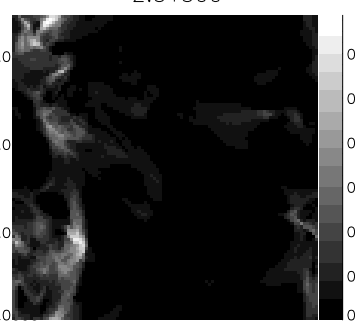

$-5.69100$

$\begin{array}{lllll}20 & 40 & 60 & 80 & 100\end{array}$
4.63100

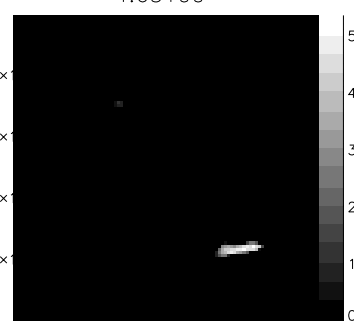

1.45500

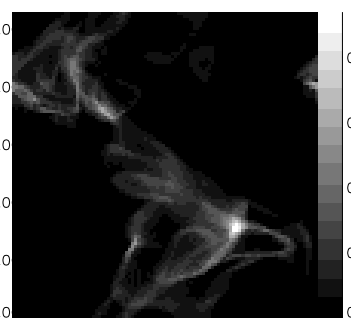

$-1.72100$

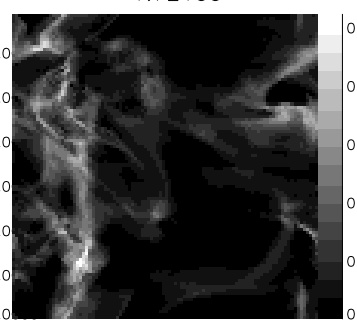

$-4.89700$

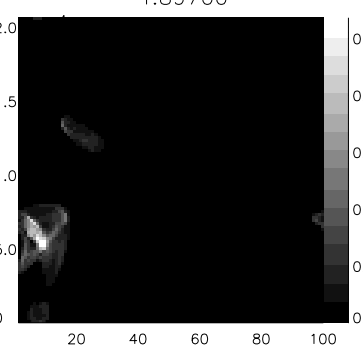

5.42500

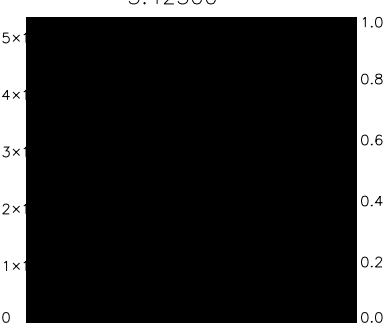

2.24900

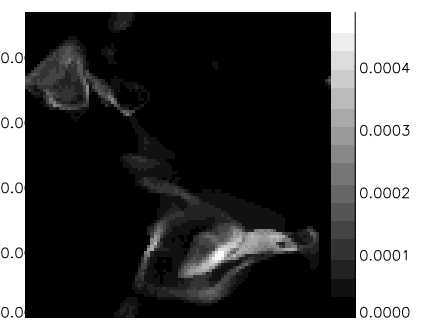

$-0.927000$

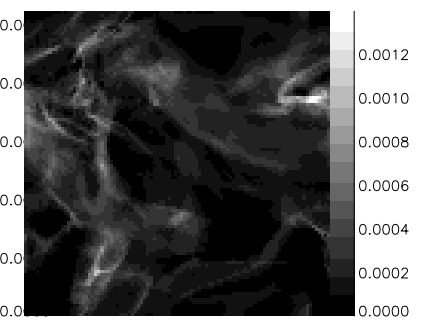

$-4.10300$

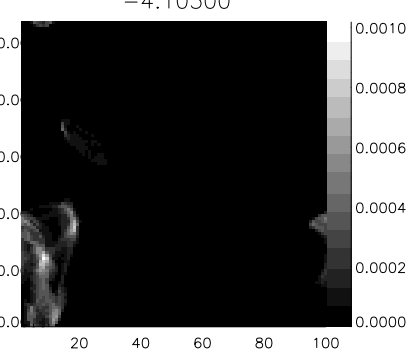

FIG. $2 b$

simulation, while for the ISM and ISM-IT runs, the forcing is turned on only after the above-mentioned conditions for star formation are met.

\subsubsection{Observing the Simulations}

In order to produce "spectra" from the simulations, we generate two types of line profiles from the simulations. In one, we simply calculate density-weighted $z$-velocity histograms (position-position-velocity or PPV cubes ). This is equivalent to assuming that the emission is optically thin, but also that the thermal broadening is negligible, so that what we see is directly the emission from each pixel at its physical velocity along the LOS. Such spectra are produced by considering all points $\left(x_{0}, y_{0}, z\right)$ over the $z$-coordinate and then creating a velocity axis by adding the contribution from each $z$-position to a velocity bin corresponding to its LOS velocity $\left(u_{z}\right)$. We refer to these as "not thermally broadened" (NTB) spectra. These synthetic observations, although unsuitable for direct comparison with real observational data because the latter cannot escape some level of thermal broadening, allow us to study the effects of velocity structure exclusively.

For the second type of spectra, we calculate the radiative transfer taking the density field in the simulation to correspond to the $\mathrm{H}$ I density and assuming LTE, optically thin lines, and Gaussian thermal broadening. In this case, every pixel "emits" at all velocities, but its emission is weighted by a Gaussian profile centered at the fluid velocity of the pixel and of a width determined by the temperature at the pixel. We will refer to this kind of spectra as "thermally broadened" (TB) spectra. In both cases "channel maps" are then produced as intensity images over the remaining two spatial coordinates ( $x$ and $y$ ) at a given $u_{z}$ interval.

In Figure 1 we show the corresponding TB and NTB spectra for nine pixels around position $(x=3, y=3)$ of the ISM run. The dotted lines represent the TB spectra, while the heavy solid lines represent the NTB spectra at low (16 velocity channels) resolution, and the light solid lines represent the NTB spectra at high-velocity resolution (64 channels). It is seen that the NTB spectra are much more irregular and spiky, because there are only 100 events in 


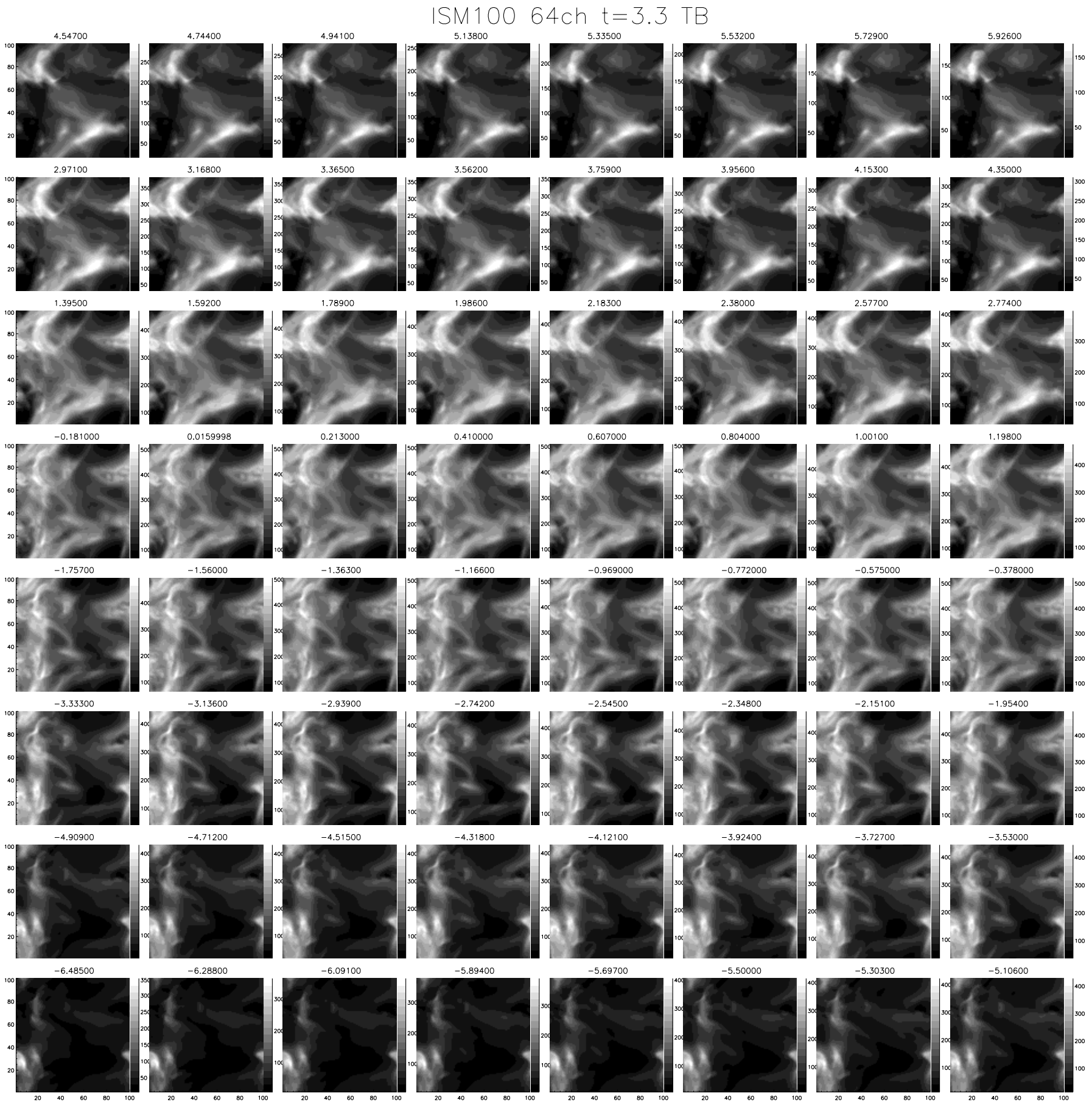

FIG. $2 c$

each LOS, which are then redistributed in either 64 (at highvelocity resolution) or 16 (at low-velocity resolution) possible bins. Note also that the spectra change substantially between the low- and high-resolution cases. In contrast, the TB spectra are much smoother, and they do not change so much with position on the POS, implying that thermal broadening "hides" the real velocity structure of the simulations. We will return to this point in $\S$ 4.1.2.

It is worth pointing out that previous works constructing velocity histograms out of three-dimensional numerical sim- ulations have frequently added up the contribution from several neighboring LOSs. For example, Falgarone et al. (1994), using $512^{3}$ simulations, considered data from a square box of $32 \times 32$ neighboring LOSs, thus including 524,288 events for each individual spectrum. However, at the risk of being velocity under-sampled, we use only one LOS per spectrum in the present work, in order to avoid further deterioration of the already low on-the-sky spatial resolution imposed by the machine available to us (a Cray Y-MP 4/64). 
ISM100 64ch $t=3.3$ NTB
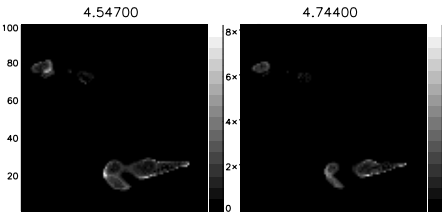

2.97100
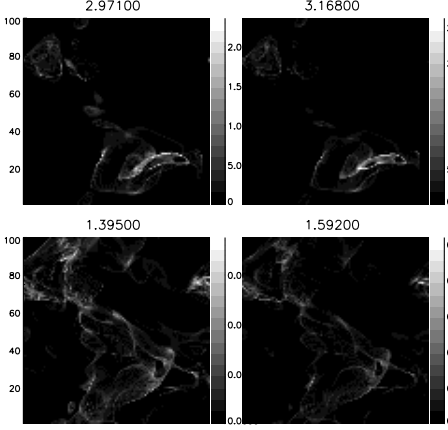

$-0.181000$

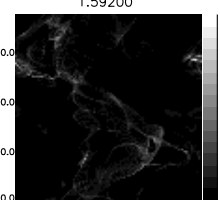

0.0159998
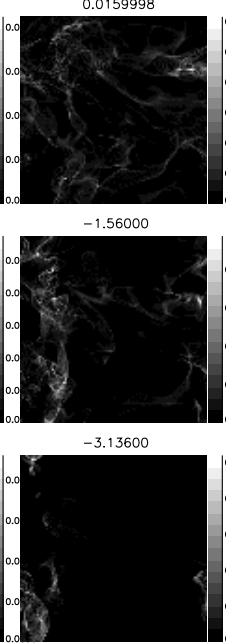

$-4.71200$

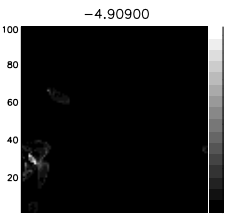

$-6.48500$

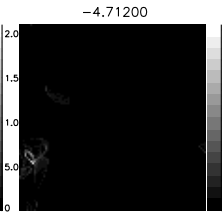

$-6.28800$
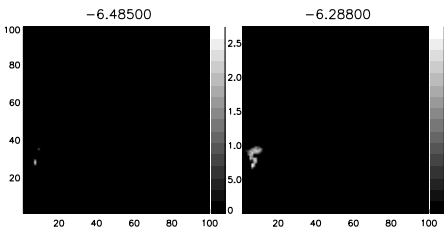

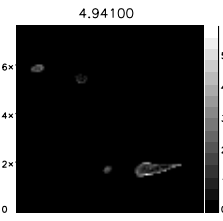

3.36500

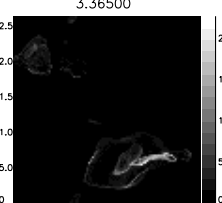

1.78900

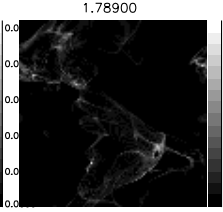

0.213000
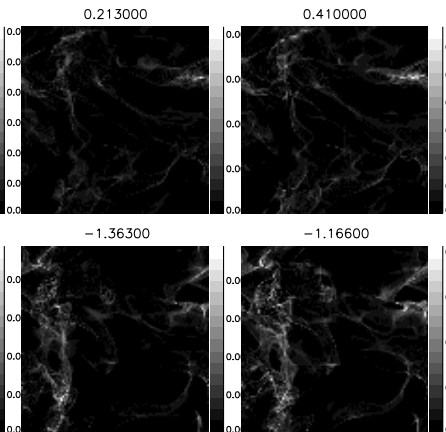

$-2.93900$

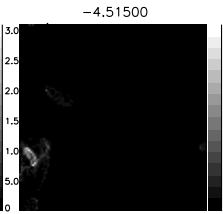

$-6.09100$

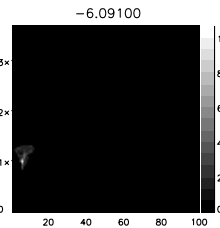

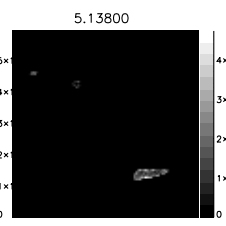

3.56200

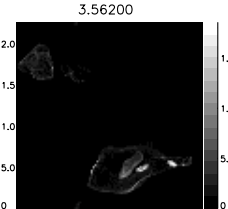

1.98600

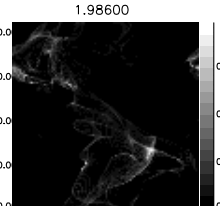

0.410000
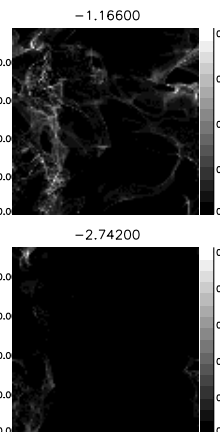

$-4.31800$
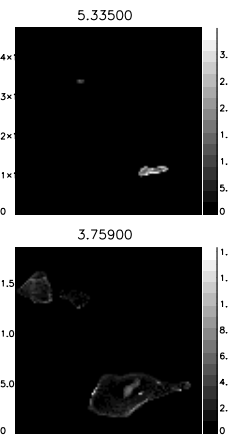

2.18300

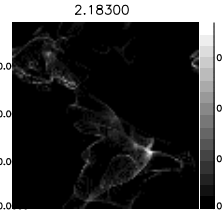

0.607000

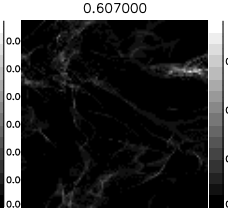

$-0.969000$
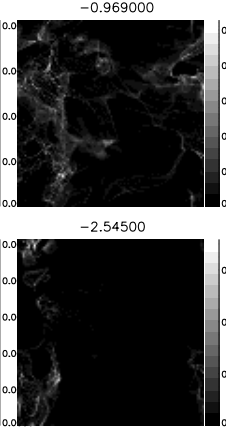

$-4.12100$

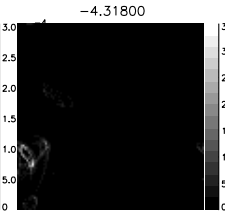

$-5.89400$
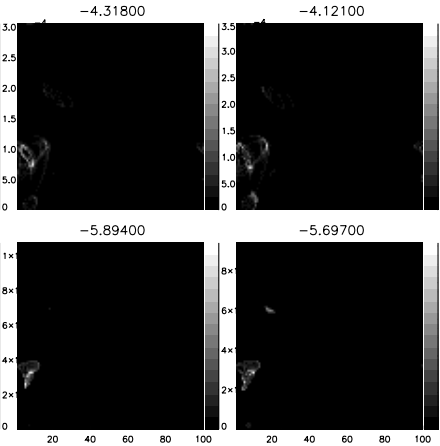

$-5.69700$

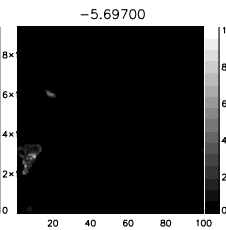

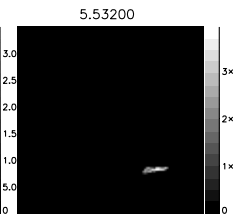
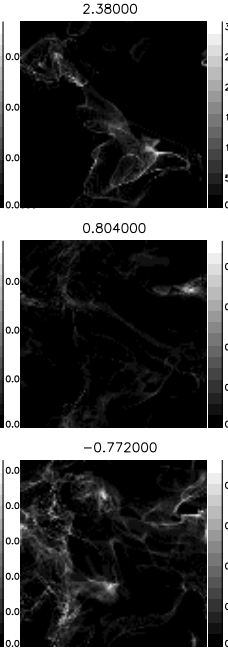

53200

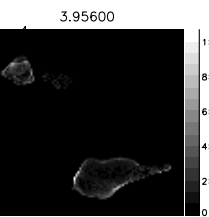

2.38000

0.804000

$-0.772000$

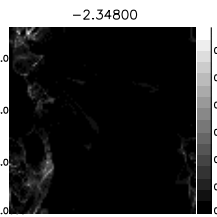

$-3.92400$

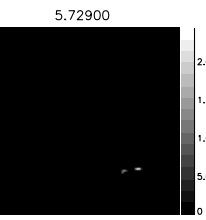

4.15300

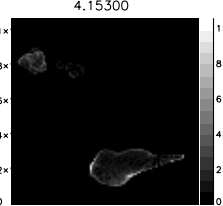

2.57700
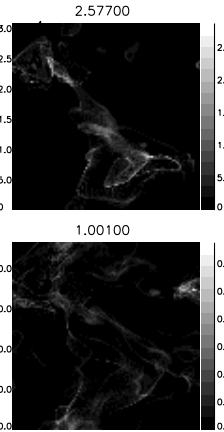

$-0.575000$
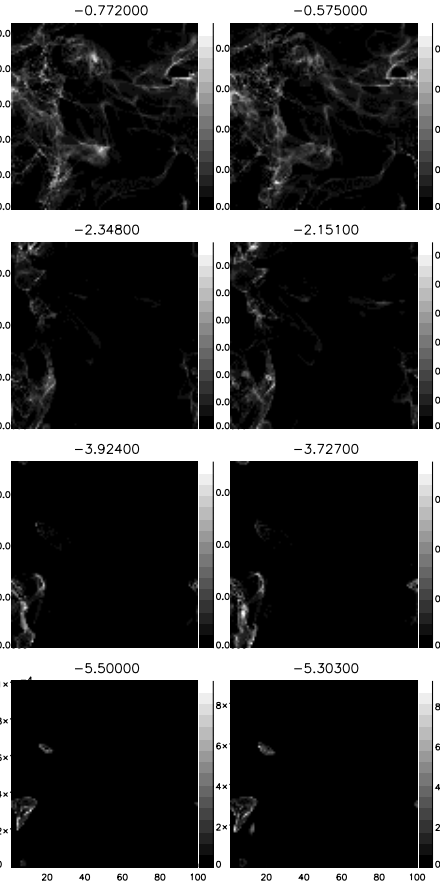

5.92600

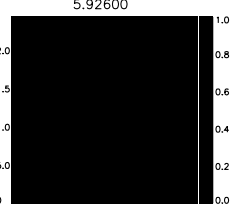

4.35000
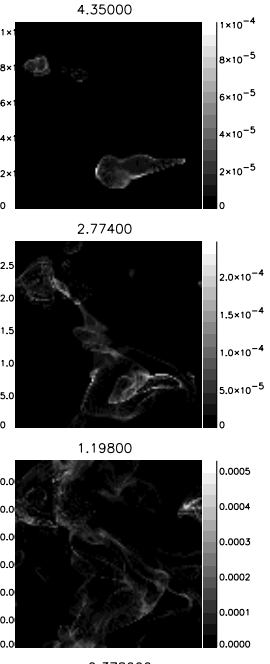

$-0.378000$
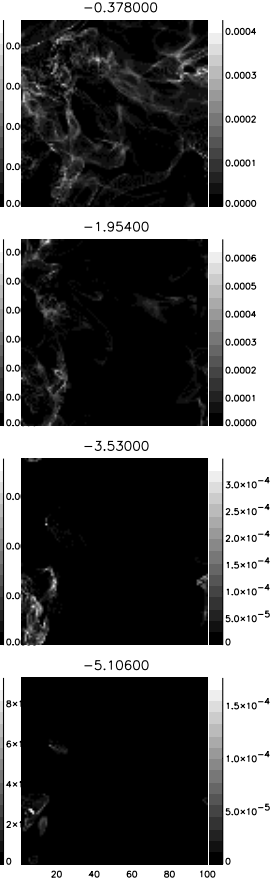

FIG. $2 d$

\subsection{Observational Data}

As the source of observational data, we use the $21 \mathrm{~cm} \mathrm{H} \mathrm{I}$ data from the Atlas of Galactic Neutral Hydrogen (Hartmann \& Burton 1997) obtained with the 25 m Leiden/ Dwingeloo telescope. Details of the observations and analysis may be found in the atlas. Here we just mention that the velocity resolution is $1.03 \mathrm{~km} \mathrm{~s}^{-1}$ and that we use the velocity range from -20 to $+20 \mathrm{~km} \mathrm{~s}^{-1}$ (the original data was taken from -450 to $400 \mathrm{~km} \mathrm{~s}^{-1}$ ).

We have chosen the north celestial pole (NCP) loop as the comparison region. This is an extended $\mathrm{H}$ I region located at

$120^{\circ} \leq l \leq 160^{\circ}, 10^{\circ} \leq b \leq 50^{\circ}$ (see $\left.\S 4.3\right)$. There are several reasons for this choice, mostly related to the fact that the ISM simulation represents a well-defined region of space, while $\mathrm{H}$ I observations suffer from confusion along the LOS. First, it is a region that lies off the Galactic plane, thus avoiding excessive contribution to the emission from very distant, physically unrelated regions. Second, the presence of a well-defined physical structure (the NCP loop) reassures us that the emission is mostly due to a well-confined region is space. Finally, there are molecular clouds and stars that allow an estimation of the distance to at least some of the molecular clouds in the region (MBM 30, 
ISM $10016 \mathrm{CH} T=3.3 \mathrm{~TB}$

S

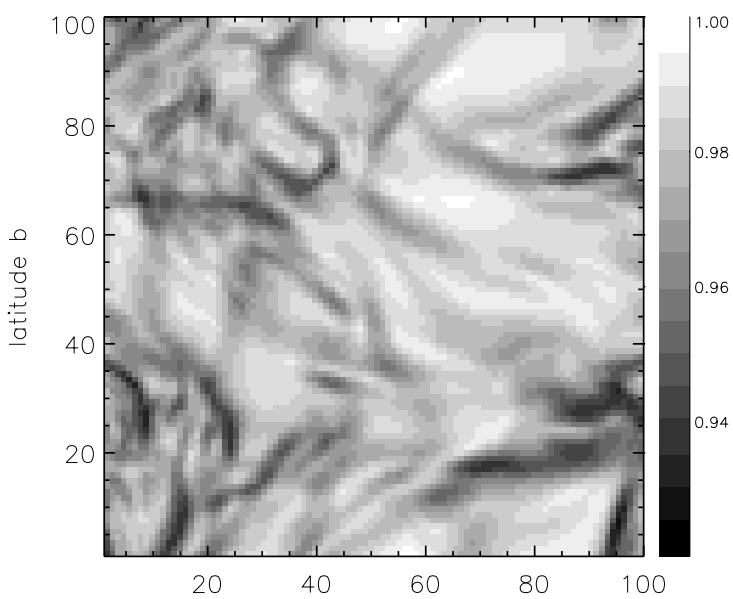

SS

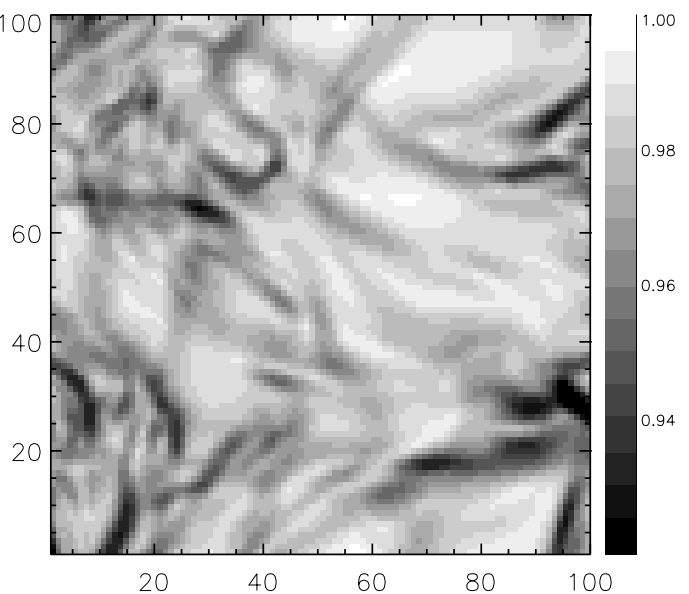

SI

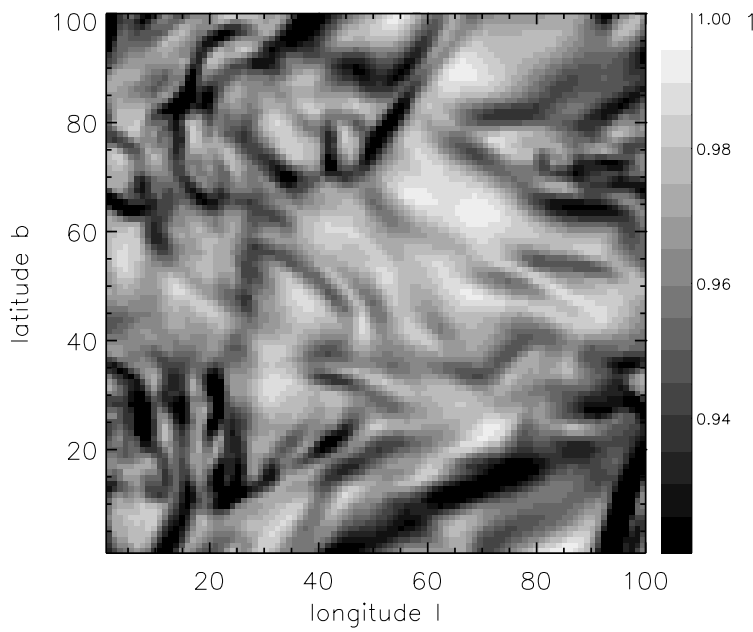

SO

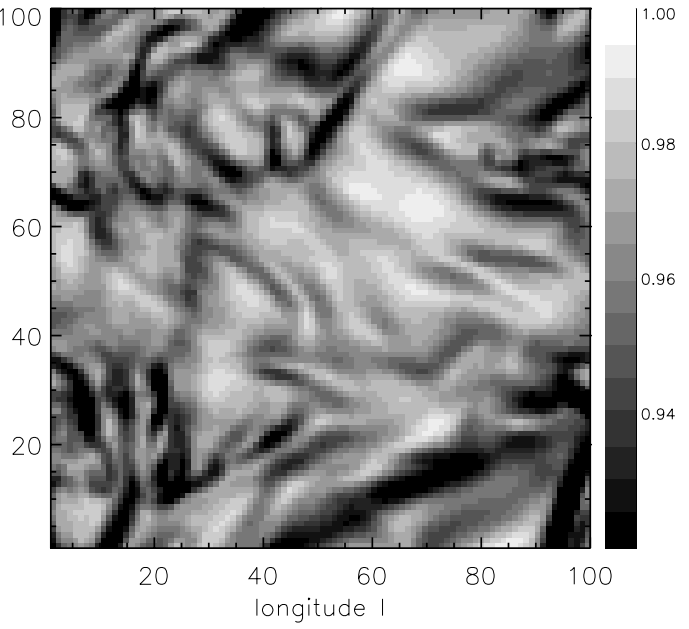

FIG. $3 a$

FIG. 3.-SCF maps for the four different "observations" of the ISM run shown in Fig. 2. Several points are noteworthy: $(a$ and $c)$ TB SCF maps highlight places where shocks and shells occur (dark regions); ( $b$ and $d$ ) NTB SCF maps exhibit a "wormy" structure probably associated with caustics in the PPV data cube; $(c)$ high resolution SCF maps from TB data do not show important differences with respect to the low-resolution TB case; $(d)$ high-resolution SCF maps from NTB data exhibit a substantially less "wormy" structure than low-resolution NTB data.

$d=110 \pm 10 \mathrm{pc})$, as well as an upper limit $(d=346 \mathrm{pc})$ to other members (MBM 29; see Penprase 1993). This implies that, if the emission lies at a distance of $\sim 230 \mathrm{pc}$ (the mean between these two extremes), the dimensions of the analyzed region are $\sim 160 \mathrm{pc}$, comparable to the physical size of the ISM simulation box.

Finally, it is worth noting that the resolution of the $\mathrm{H} \mathrm{I}$ data changes with Galactic latitude: it is 0.5 for $b \leq 35^{\circ} ; 0^{\circ} .6$ for $35^{\circ} \leq b<45^{\circ}$; and 0.7 for $45^{\circ} \leq b<55^{\circ}$. This has the effect of slightly overestimating the SCF at high latitudes, since we assume a uniform grid spacing of 0.5 .

\section{THE SPECTRAL CORRELATION FUNCTION}

A detailed discussion of the development and advantages of the SCF is given by RGWW. For completeness we repeat the necessary definitions here, give a physical interpretation of the SCF, and then discuss the strategies for its application and the effects of noise.

\subsection{Definitions and Physical Interpretation}

Given two arbitrary spectra with antenna temperatures $T_{1}(v)$ and $T_{0}(v)$, the SCF is defined by RGWW as

$$
\mathrm{SCF} \equiv 1-\sqrt{\frac{\int\left[s T_{1}(v-l)-T_{0}(v)\right]^{2} d v}{s^{2} \int T_{1}^{2}(v-l) d v+\int T_{0}^{2}(v) d v}},
$$

where $l$ and $s$ are free parameters chosen to minimize the integral $\Upsilon=\int\left[s T_{1}(v-l)-T_{0}(v)\right]^{2} d v$. Thus, for two identical spectra with infinite signal-to-noise $(\mathrm{S} / \mathrm{N})$ ratio, $\mathrm{SCF}=1$, while for two completely uncorrelated spectra, $\mathrm{SCF}=0$.

Given the parameters $l$ and $s, \mathrm{RGWW}$ define four modes of the SCF: (1) $\mathrm{SCF}^{0} \equiv \operatorname{SCF}(s=1, l=0)$, which compares the two spectra in their raw form. Values of $\mathrm{SCF}^{0}$ close to unity imply that the spectra resemble each other in shape, amplitude, and velocity offset. (2) $\mathrm{SCF}^{l} \equiv \operatorname{SCF}(s=1, l)$, with $l$ chosen to minimize $\Upsilon$. This form eliminates differen- 
S

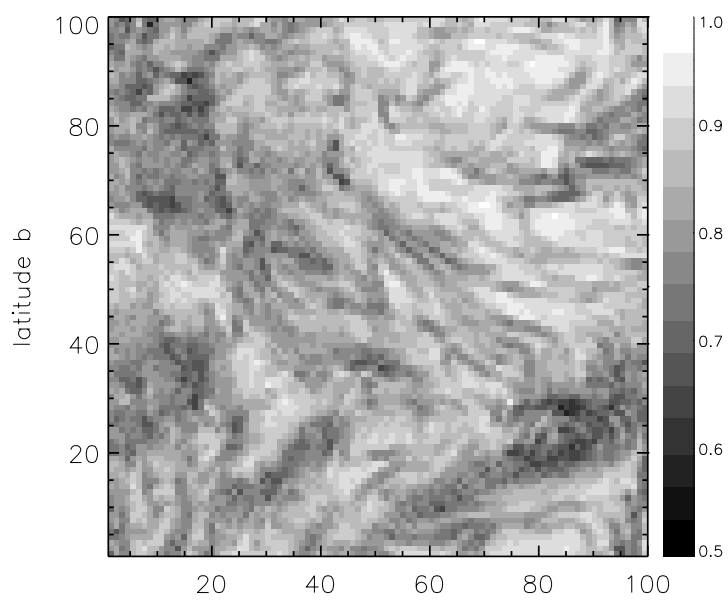

Ss

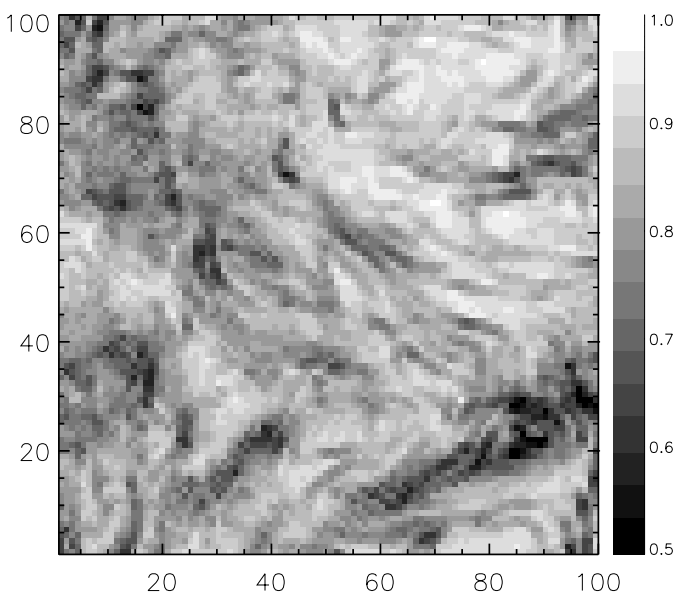

S!

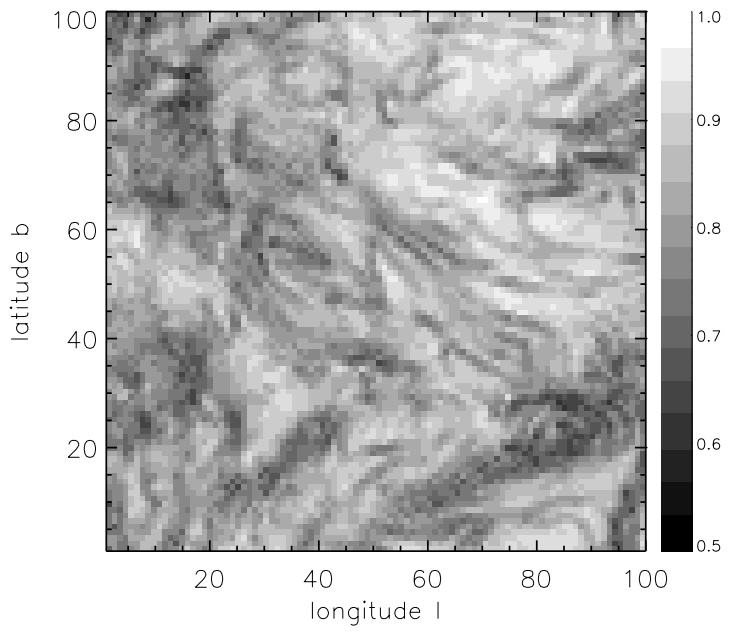

so

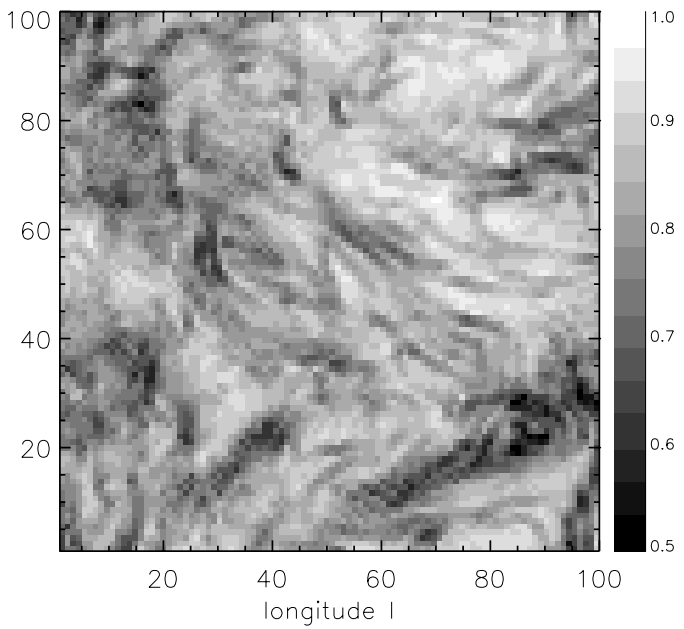

FIG. $3 b$

ces between the spectra arising from pure velocity offsets. Values of $\mathrm{SCF}^{l}$ close to unity imply that the spectra resemble each other in shape and amplitude, regardless of velocity offset. (3) $\mathrm{SCF}^{s} \equiv \operatorname{SCF}(s, l=0$ ), with $s$ chosen to minimize $\Upsilon$. This form eliminates differences between the spectra arising from pure intensity scalings. Values of $\mathrm{SCF}^{s}$ close to unity imply that the spectra resemble each other in shape and velocity offset, regardless of absolute amplitude. (4) $\mathrm{SCF}^{l, s} \equiv \operatorname{SCF}(s, l)$, with both $s$ and $l$ chosen to minimize $\Upsilon$. This is then the general definition of the SCF (eq. [1]) and eliminates differences arising from both velocity offset and amplitude. In this case, values close to unity imply that the two spectra resemble each other in shape only.

\subsection{Effects of Instrumental Noise}

As has been shown by RGWW (see also Padoan, Rosolowsky, \& Goodman 2001, hereafter PRG), the SCF can be strongly affected by noise, and these effects have to be evaluated. PRG propose to "clean" the effects of the noise by dividing the actual value of the SCF by an "optimal value" of the SCF, i.e., by the maximum value that the SCF would have if the only difference between both spectra were the random noise. The optimal value is given by (see PRG)

$$
\operatorname{SCF}(Q)=1-\frac{1}{Q}
$$

where $Q$ is the "spectrum quality" defined as

$$
Q_{i}=\frac{1}{\sigma} \sqrt{\frac{\sum_{v} T_{i}^{2} \delta v}{N_{\mathrm{ch}} \delta v}}=\frac{1}{\sigma} \sqrt{\left\langle T_{i}^{2}\right\rangle},
$$

where in turn $\sigma$ is the mean rms noise, $N_{\mathrm{ch}}$ is the number of velocity channels, $\delta v$ is their width, and $\left\langle T_{i}^{2}\right\rangle$ is the mean value of the emission in the velocity interval. As can be seen, for high values of the $\mathrm{S} / \mathrm{N}$, the parameter $Q$ goes to infinity, and the SCF is renormalized by a number close to unity.

PRG show that adding noise to both the observed (molecular, with $\mathrm{S} / \mathrm{N}$ values of the order of a few) and the synthetic (without intrinsic noise) spectra until the $\mathrm{S} / \mathrm{N}$ is constant throughout the data and renormalizing the SCF as indicated in equation (2) gives values of the SCF essentially independent of the noise level. 
S

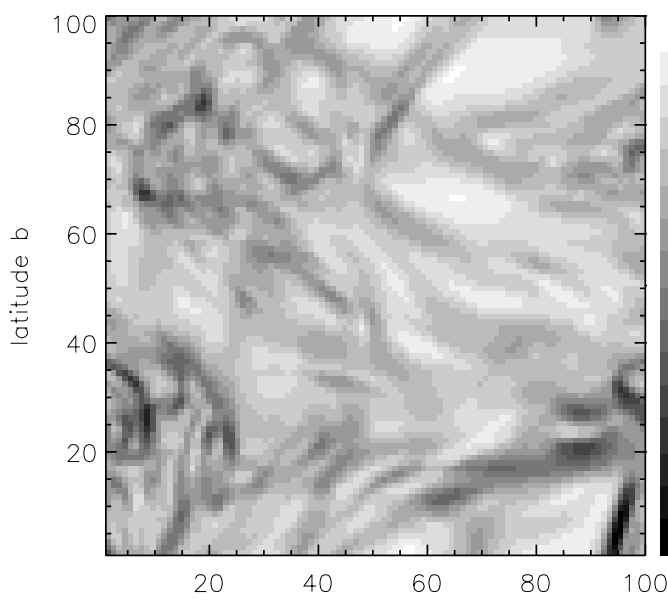

SI

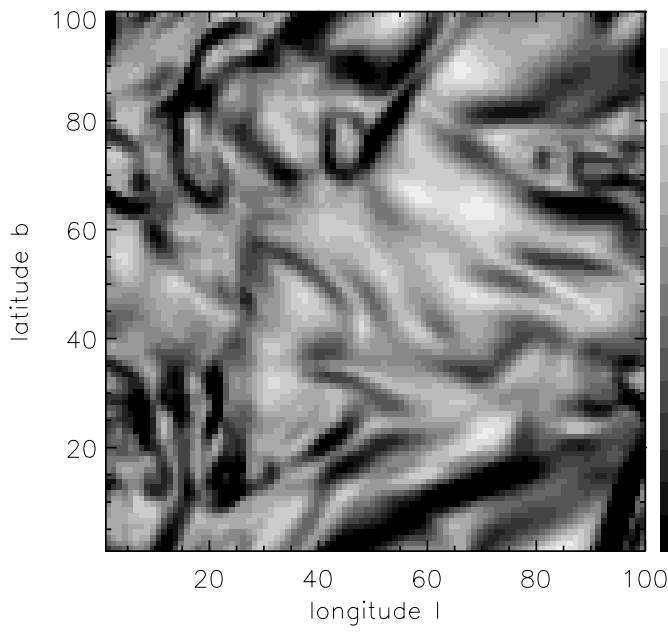

SS

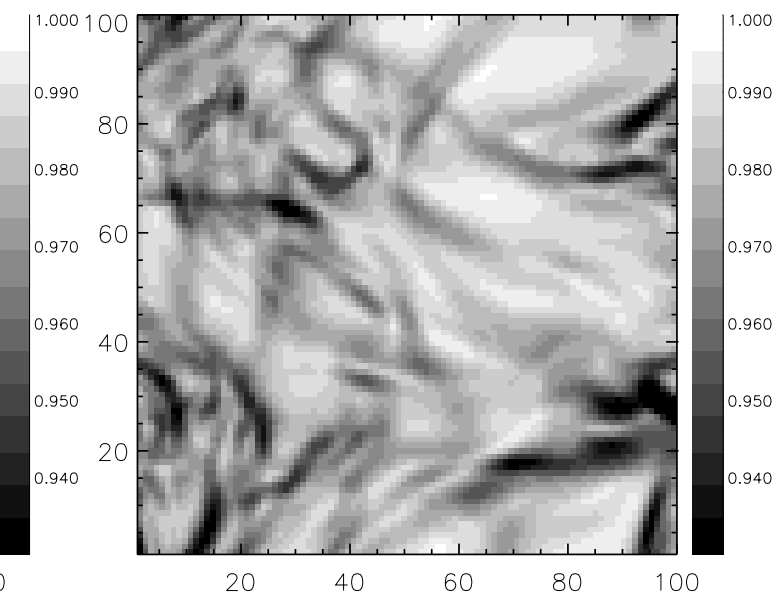

SO

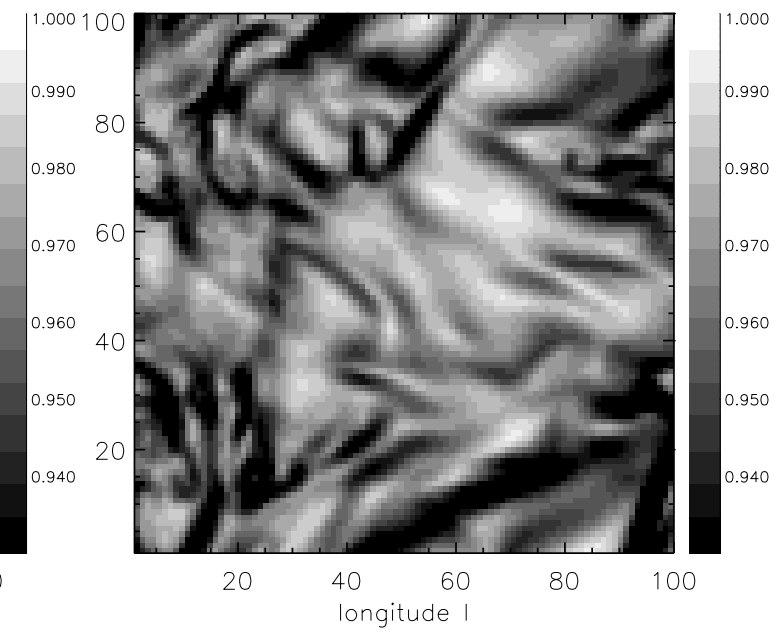

FIG. $3 c$

In the present work we deal with data that are almost unaffected by noise: on the one hand, the numerical simulations are intrinsically noiseless; on the other, for the $\mathrm{H}$ I data (Hartmann \& Burton 1997; see $\S 2$ ) the characteristic value of $\sigma$ is $0.07 \mathrm{~K}$, and its characteristic antenna temperature value is of several $\mathrm{K}$, suggesting high values of $Q$. Indeed, we have verified that for the observed data set ( $\mathrm{H} \mathrm{I}$ ) used here, the mean value of $Q$ is 98.8 (see $\S 4.3$ ). Therefore, in this paper we do not correct for the effects of noise.

\subsection{Velocity Window}

In order to compute the four kinds of the SCF, in particular, the modes $\mathrm{SCF}^{l, s}$ and $\mathrm{SCF}^{l}$, which imply shifting one spectrum with respect to the other, RGWW use velocity channels within three FWHMs of the velocity centroid, with the FWHM and the velocity centroid computed by fitting a Gaussian to the line profiles. In turn, PRG use a $10 \Delta_{2} v$ velocity window, where $\Delta_{2} v$ is the standard deviation of the velocity of a spectrum created by averaging over the whole map. However, in our case of negligible noise, we simply extend the original data sets (observational and synthetic) by adding zeros on both sides of the spectral lines to produce a spectral window 3 times wider than the original.

\subsection{Maps and Histograms of the SCF}

The SCF, like the regular autocorrelation function, is in general a function of spatial separation, and this aspect of it is discussed by PRG. However, RGWW has used it in a "local" mode, which we follow here. In this form, we construct maps of the average value of the SCF in boxes of 3 pixels per side in a given spectral map. That is, to each point on the $(x, y)$-plane (the POS of the simulations) we assign the average value of the SCF evaluated using that point and each of its eight nearest neighbors. Note that with this approach, the SCF maps are useful for exhibiting smallscale spatial variations in the line spectra. In addition, in order to quantify the similarity between spectral maps, RGWW also considered the distribution (histogram) of the $\mathrm{SCF}$ in a given map and its moments, and we follow this approach here.

In practice, the nearest-neighbor variant of the SCF used here acts like an angle-average of the spatial (i.e., in the 
$S-$

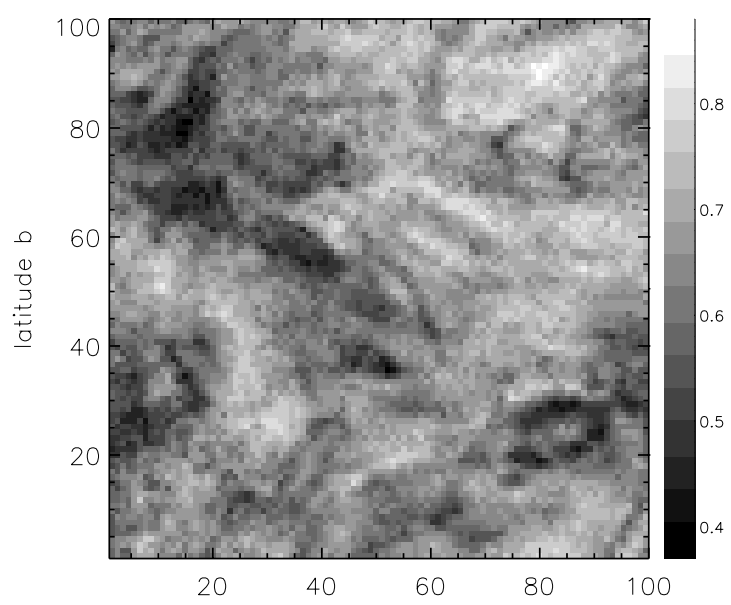

SI

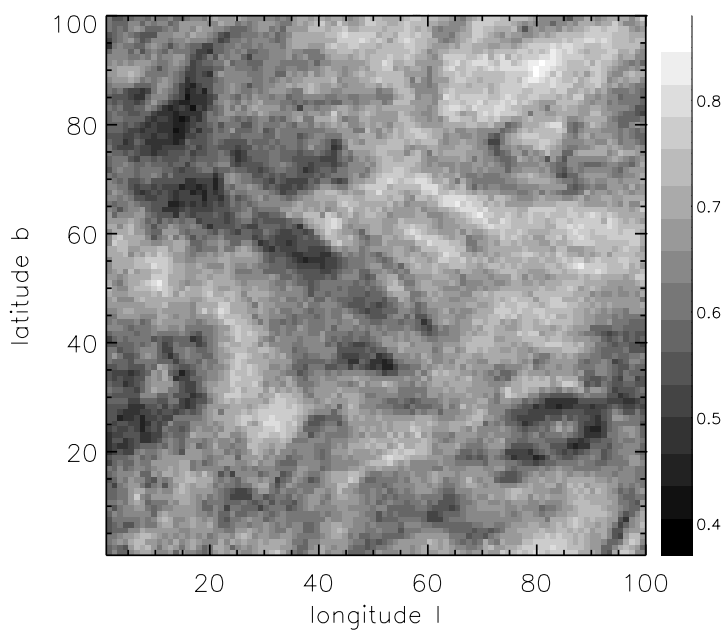

SS

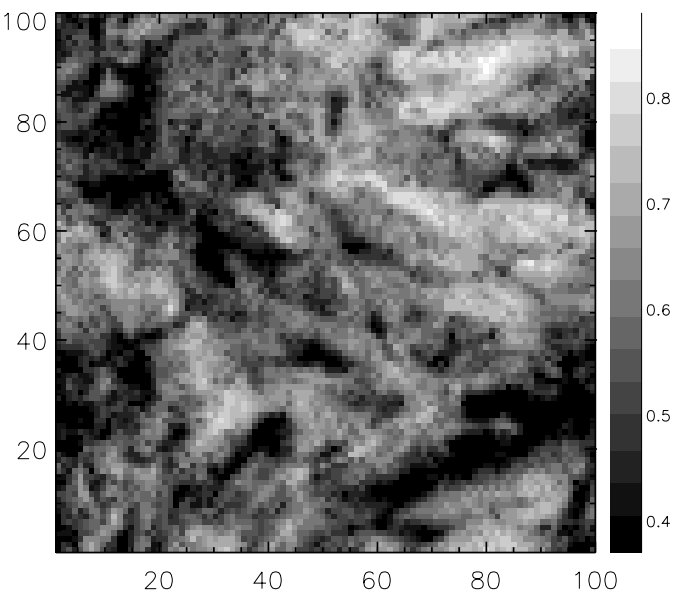

SO

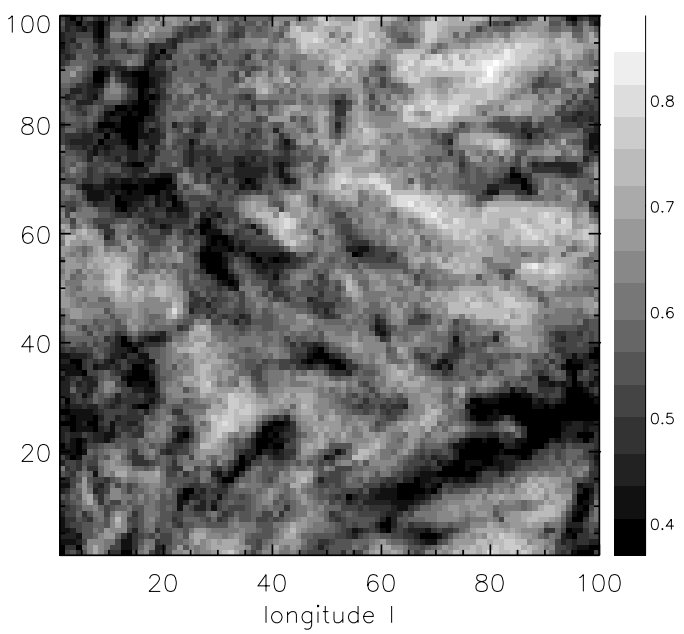

FIG. $3 d$

POS) derivative of the spectral profiles. Sites of strong profile variation appear in the SCF maps as low values (dark regions), and soft variations appear as large values (light regions). That is, the SCF stresses small-scale changes (on the POS) in the line spectra. Moreover, the fact that the SCF considers the whole velocity structure in the line profiles at each position, implies that maps of the SCF give more information than the channel maps alone, because they make velocity variations (as well as density ones) evident to the eye, which might escape examination of pairs of channel maps. The SCF histogram moments then quantify such velocity and intensity differences.

\section{RESULTS AND INTERPRETATION}

\subsection{Understanding the $S C F$}

In order to "calibrate" the SCF and to determine its response to changes in the spectral resolution and thermal broadening, we have simulated four different "observations" of the ISM run, labeled 16-TB, 16-NTB, 64-TB, and 64-NTB, where the number indicates the number of velocity channels, and the letters TB/NTB indicate whether or not thermal broadening was included. We also have produced another set (TB and NTB) of "observations" of this run, but with the velocity artificially multiplied by a factor of 6 , in order for the line spectra and the SCF histograms to better match those of the NCP loop. This will be discussed in $\S 5.2$. Velocity channel maps, maps of the SCF, and SCF histograms are shown in Figures 2, 3, and 4, respectively. Each case (16/64 velocity channels and TB/NTB) is indicated at the top of each figure.

\subsubsection{Effects of the Velocity Resolution}

Comparing the NTB channel maps (Figs. $2 b$ and $2 d$ ), we note that at higher velocity resolution the small-scale structure is more prominent, a fact already noted by Pichardo et al. (2000), and interpreted by W. Henney, E. Vázquez-Semadeni, \& B. Pichardo (2002, in preparation) in terms of the presence of "caustics" or "cusps" (loci of high intensity due to velocity crowding) in the PPV data cube. We will return to these caustics in $\S 5.1$. However, this effect is almost nonexistent in the more realistic TB case. 


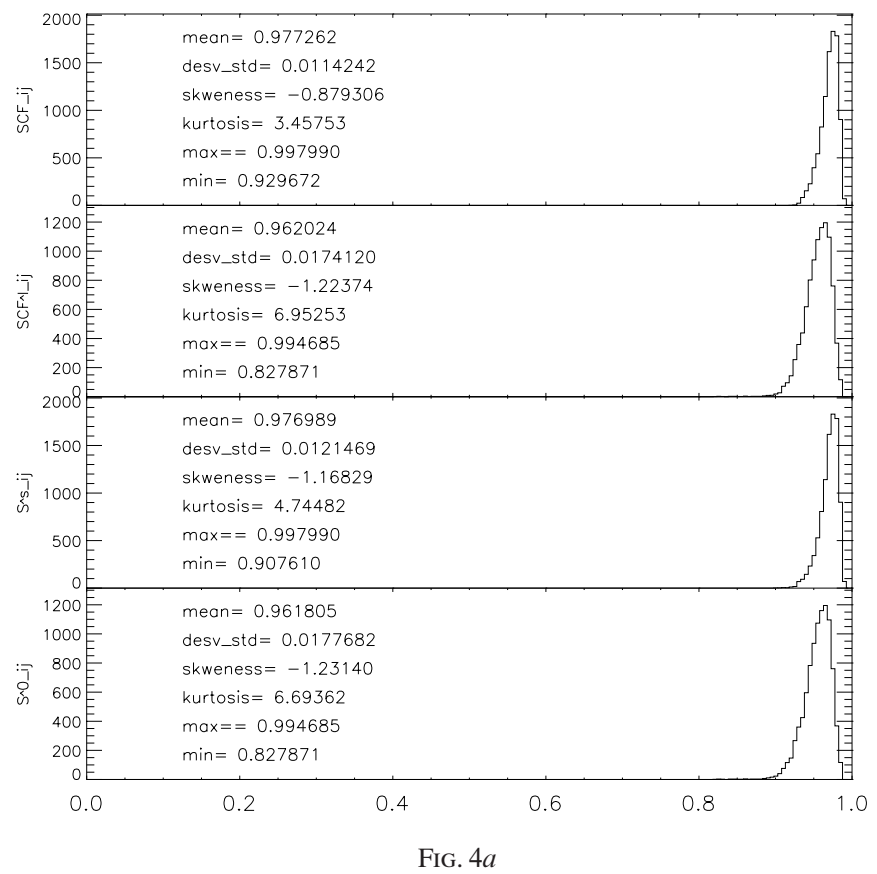

ISM $10064 \mathrm{ch} \mathrm{t}=3.3 \mathrm{~TB}$

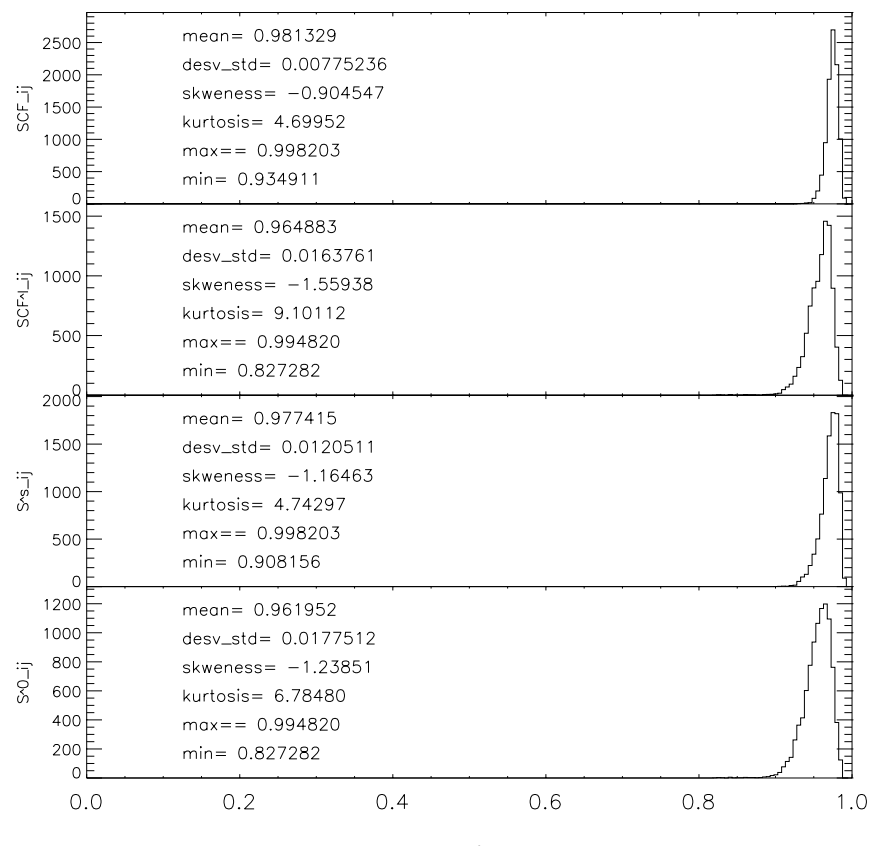

FIG. $4 c$

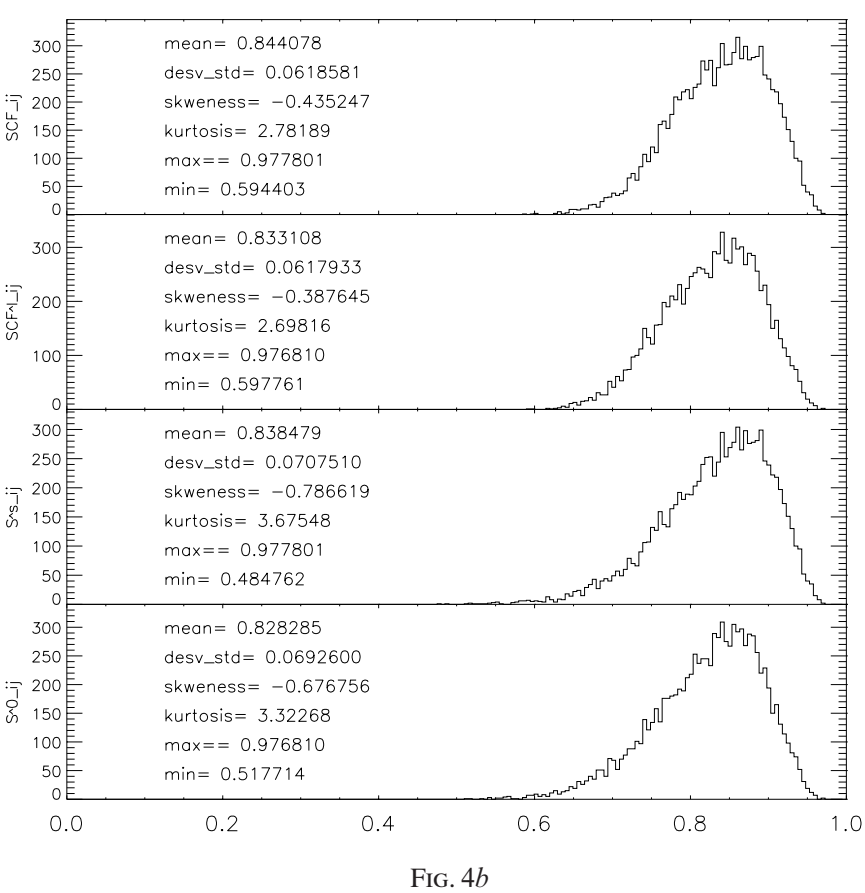

ISM100 64ch $\mathrm{t}=3.3$ NTB

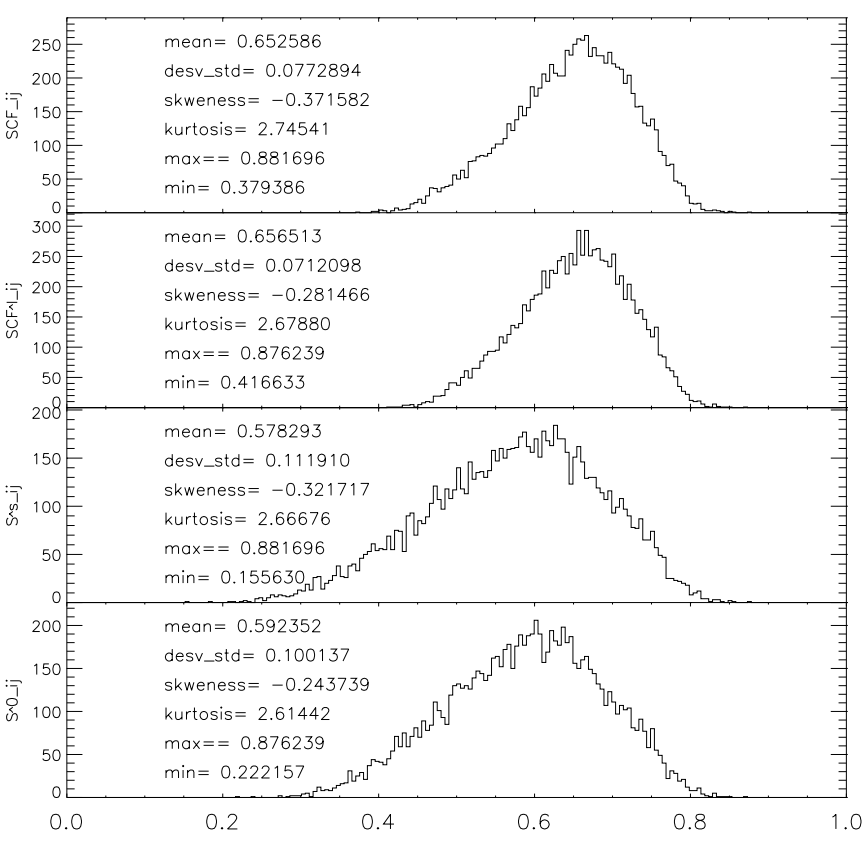

FIG. $4 d$

FIG. 4.-Histograms of the SCF maps shown in Fig. 3. Since the correlation between neighboring spectra is larger in the TB case than in the NTB one, the histograms for the former have larger mean values and smaller widths and skewness values than the latter. Also, a high velocity resolution in the absence of strong thermal broadening reduces the correlation, giving wider histograms with lower means.

Additionally, it can be seen that the NTB map at low velocity (or "spectral") resolution (Fig. 3b) exhibits a clearly "wormy" or "stringy" structure, probably related to the caustics mentioned above $(\S 5.2)$. However, the wormy structure is almost absent from the high-resolution SCF maps (Fig. $3 d$ ). This is an artifact of the low spatial resolution of our simulations (100 grid points per dimension), which causes any given point in the simulated POS to have very few points along the LOS contributing to the intensity in any one velocity channel (less than two on average at a velocity resolution of 64 channels), in turn causing the line profiles to be highly irregular (see Fig. 1). Thus, what little signal is available is diluted over many velocity channels, and only the most prominent features are preserved, with a 
ISM-IT 16ch $t=1.4$

12.0000

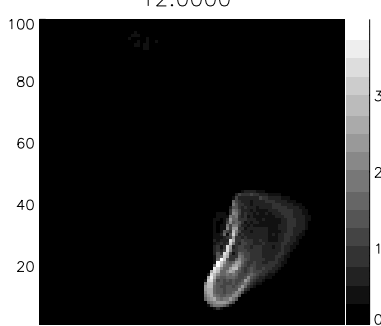

8.00000

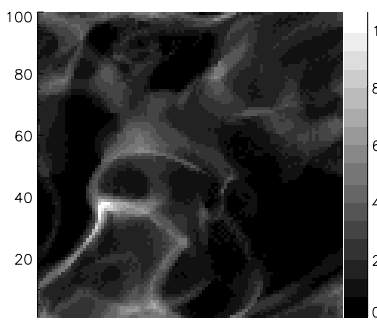

4.00000

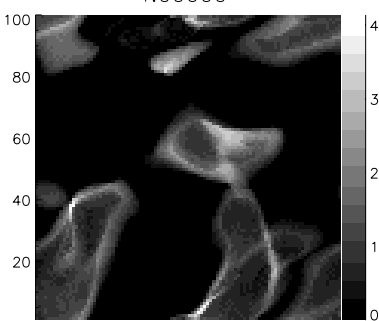

0.00000

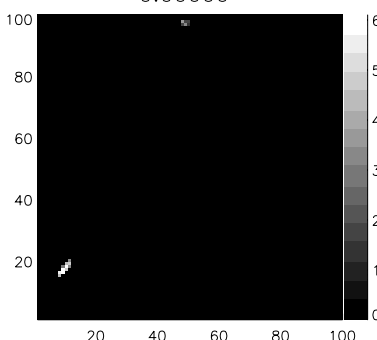

13.0000

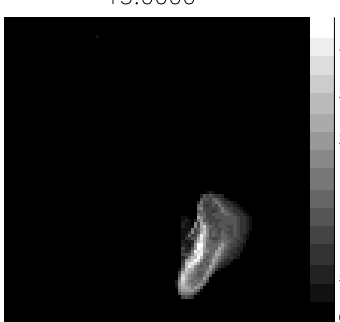

9.00000

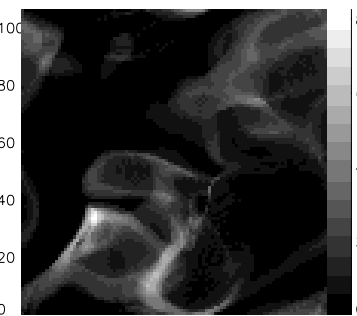

5.00000

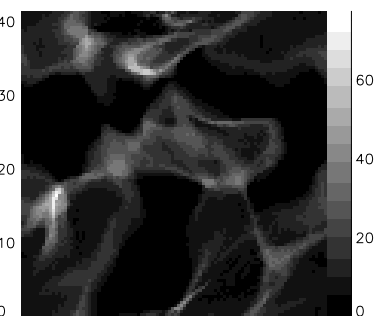

1.00000

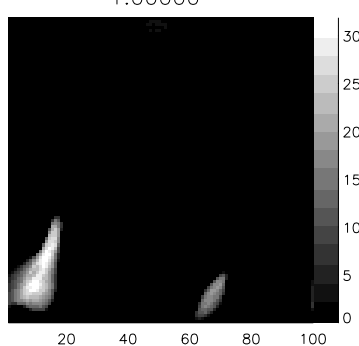

14.0000

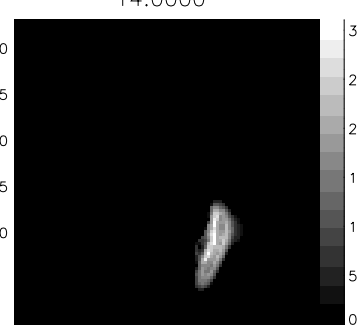

10.0000

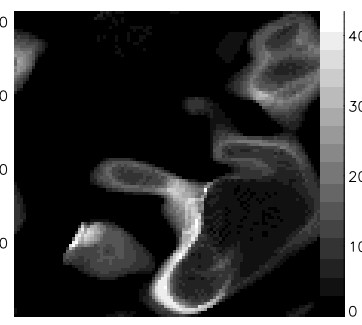

6.00000

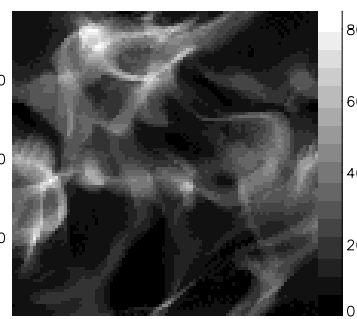

2.00000

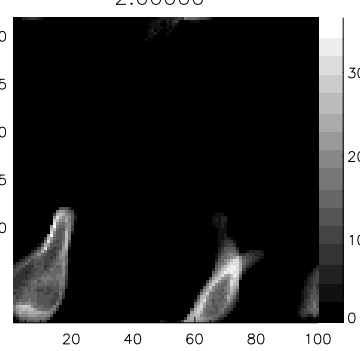

15.0000

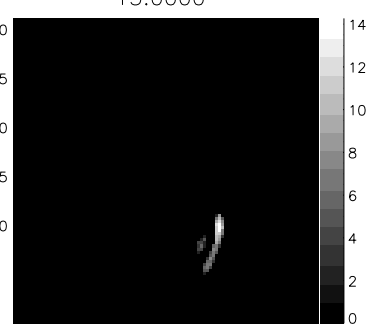

11.0000

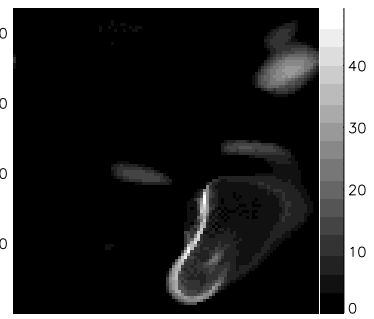

7.00000

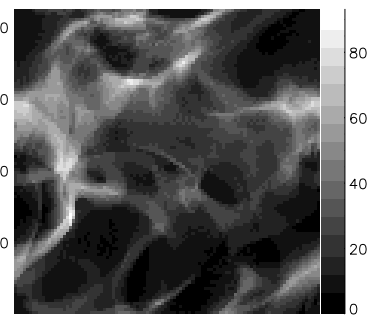

3.00000

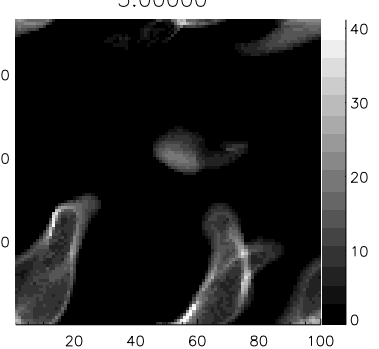

FIG. $5 a$

FIG. 5.-NTB channel maps for (a) the isothermal, self-gravitating run (ISM-IT) with pointlike energy; (b) the isothermal, purely hydrodynamic run (IT)

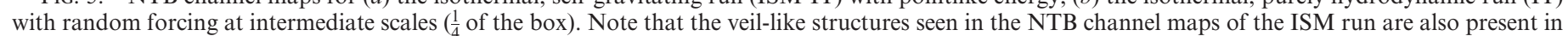
these simulations, but not so the very large-scale structures (of the size of the box) seen in that run.

very "blurred" quality. This shows that the effective velocity resolution is in effect limited by the spatial resolution. The scattered character of the NTB line profiles at high velocity resolution is also evident in their corresponding SCF histograms (Fig. 4d), which are clearly much wider and have lower mean values than the low-resolution ones (Fig. $4 b$ ), indicating that the profiles vary strongly among neighboring LOSs.

\subsubsection{Effects of Thermal Broadening}

We now turn to the effects of thermal broadening on velocity channel and SCF maps and on the SCF histograms. For the discussion, we consider only the low velocity resolution observations because of the poor statistics present in the high-resolution ones.

It is first important to note that the ISM run contains large regions occupied by warm $\left(T \sim 1.5 \times 10^{3} \mathrm{~K}\right)$ gas, whose thermal velocity dispersion dominates the line profiles at any given position on the POS, as seen in Figure 1. This is because, even though the low-temperature regions (clouds) contain supersonic motions, these motions are subsonic compared to the sound speed of the warm medium. Moreover, the warm gas is barely transonic with respect to itself. Thus, the thermal broadening due to the warm gas along the LOS masks the bulk velocity structure seen in the profiles, which are nearly perfectly Gaussian in the TB case, in contrast with the extremely irregular profiles seen in the NTB case. This effect is expected to occur in actual observations of multitemperature atomic gas, so that the velocity structure of the cold $\mathrm{H}$ I gas may only be seen in absorption (Gibson et al. 2000; Heiles 2001).

This effect is also seen in the channel maps. The structure in the NTB channel maps, regardless of resolution, can be described as having the appearance of superposed veils, with sharp, bright edges delimiting fainter regions. In con- 


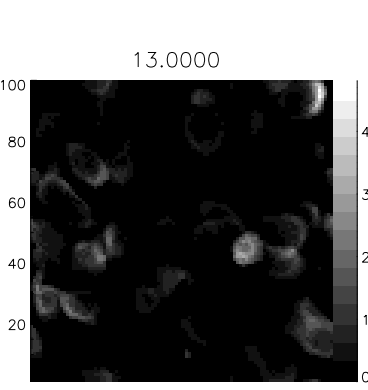

9.00000

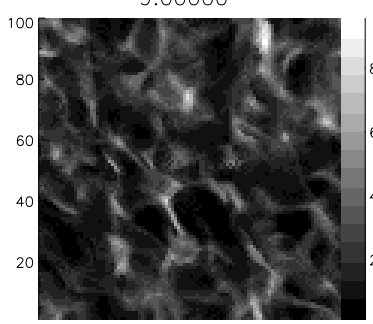

5.00000

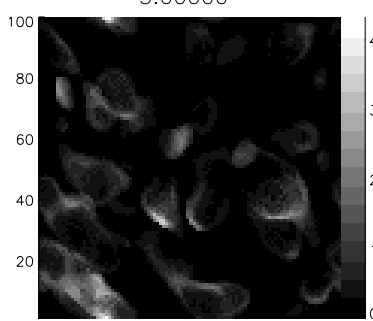

1.00000

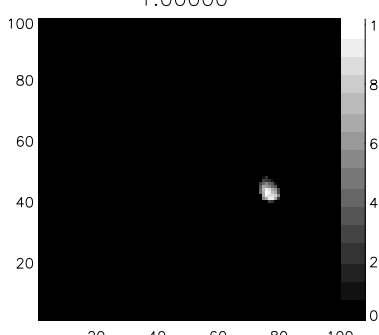

14.0000

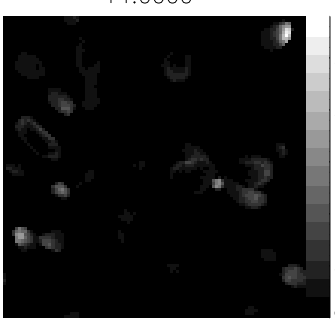

10.0000

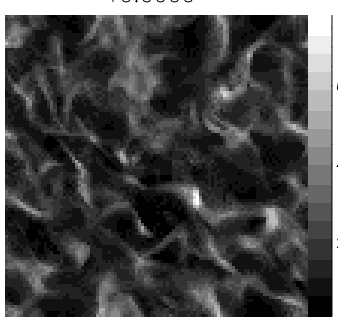

6.00000

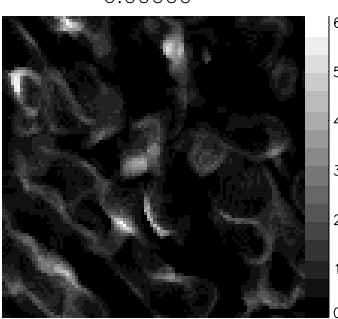

2.00000

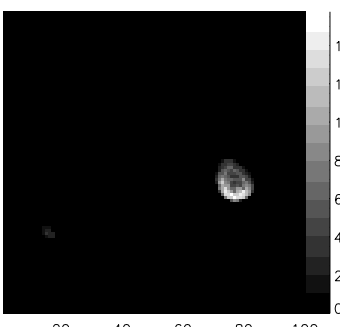

15.0000

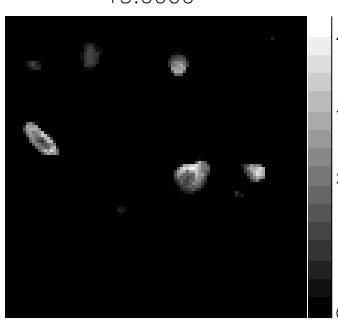

11.0000

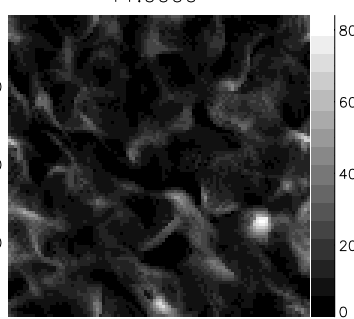

7.00000

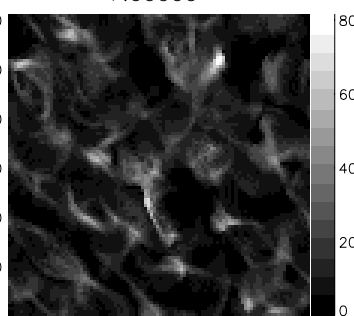

3.00000

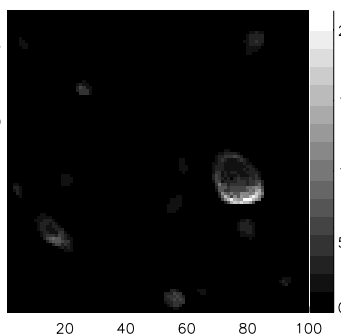

16.0000

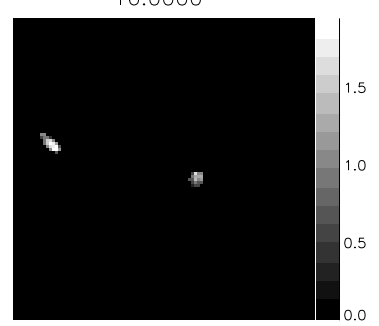

12.0000

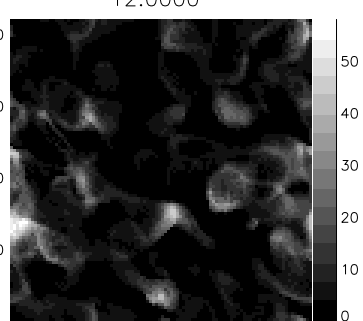

8.00000

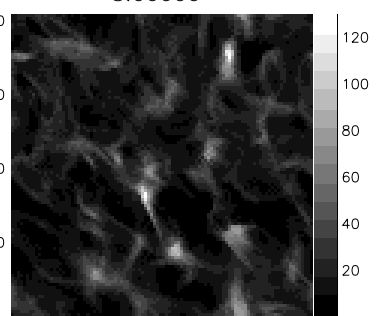

4.00000

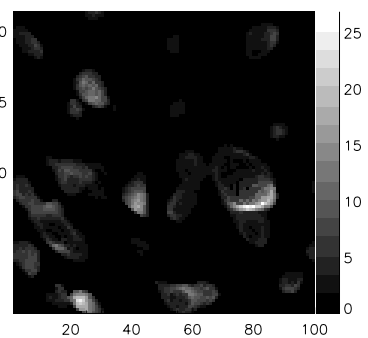

FIG. $5 b$

trast, the TB channel maps are much smoother, losing the veil-like appearance and becoming more similar to maps of the total integrated intensity. Thus, only the gross features, such as large-scale velocity gradients, remain noticeable. Again, this effect is also expected to occur in actual observational data of warm gas.

In the SCF maps, the "wormy" nature of the NTB cases, mentioned in $\oint 4.1 .1$, is seen to lose its small-scale parts in the TB maps. That is, the "worms" in the NTB case appear to "bundle up" into larger-scale filaments, in a sort of self-similar or hierarchical manner. However, in the TB SCF maps, only the bundles appear to be preserved, while their smaller constitutive filaments are not seen. As will be discussed in $\S 5$, the small-scale filaments, if originated by the caustics in the PPV cube, would be an artifact of the velocity segregation of the data on the process of a spectroscopic observation. Thus, thermal broadening has the fortunate effect of counteracting this spurious small-scale generation that is caused by the velocity separation effected by the observation process.
Another important feature is that the SCF maps for the TB observations have a much smaller dynamic range than the NTB ones, indicating a much lower variability of the $\mathrm{SCF}$ in the former case, although, on the other hand, the same overall structures are seen in both types of maps. The SCF histograms clearly reflect the above effects. While the histograms of the NTB observations have large variances (Fig. 4b) and moderate mean values, those for the TB (Fig. $4 a$ ) cases are much narrower and with means very close to unity, the maximum possible value of the correlation. The small variances and large means of the TB cases again indicate the "erasure" of the velocity structure by thermal broadening, which causes all spectra to be much more similar to each other than in the NTB cases. Furthermore, this indicates that the structure seen in the TB SCF maps should be due mostly to variations in intensity rather than in velocity structure. This is, indeed, confirmed by the fact that in the TB cases the histograms of $\mathrm{SCF}^{s}$, which can compensate for intensity variations but not for velocity shifts, are much tighter (have smaller variances) than those of $\mathrm{SCF}^{l}$, which compensates in the opposite manner. Instead, in the NTB 


\section{ISM-IT $16 \mathrm{ch} t=1.4$}

S

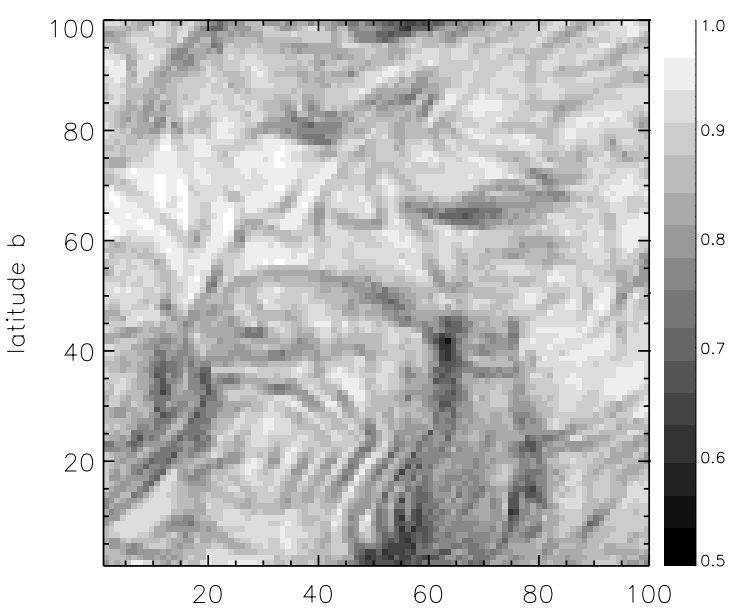

SI

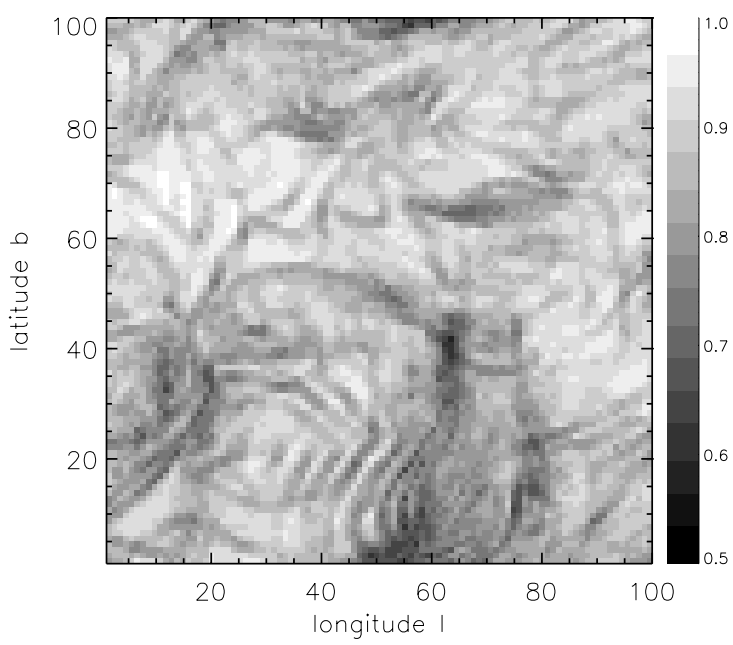

SS

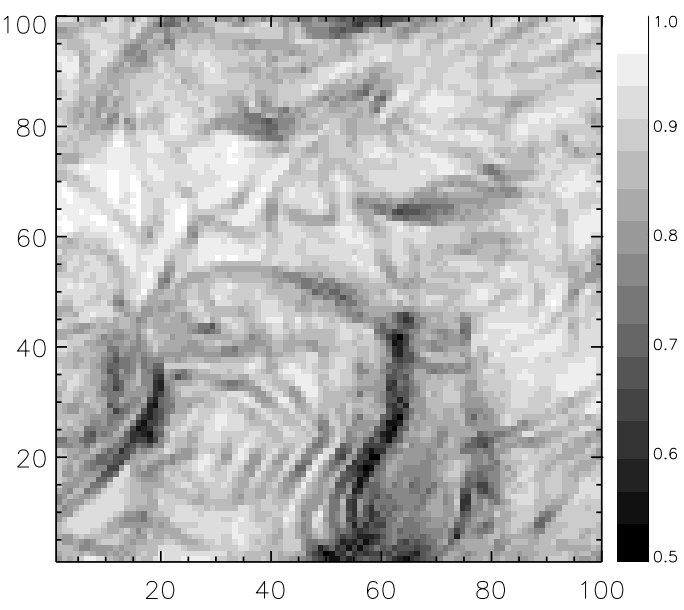

so

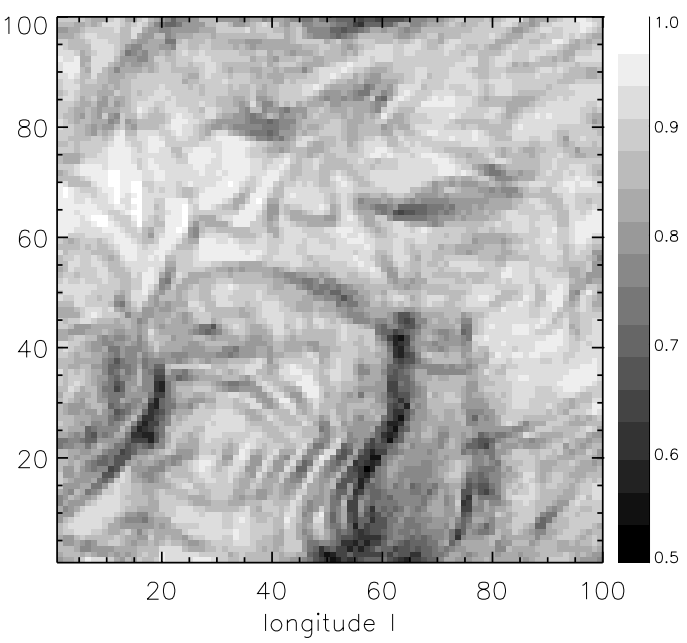

FIG. $6 a$

FIG. 6.-SCF maps for Fig. 5. The wormy structure seen in the ISM run is also present but bundling into more roundish forms, following the structures that appear in the channel maps (Fig. 5).

cases, the histograms of $\mathrm{SCF}^{s}$ have comparable (in the low velocity resolution case) or even larger (in the highresolution case) variances than the histograms of $\mathrm{SCF}^{l}$, indicating that in this case the variability in the maps is due mainly to the velocity structure.

\subsection{Comparison between Numerical Simulations}

We now compare these results with two other numerical simulations. As mentioned in $\S 2.1$, we consider an isothermal simulation including the magnetic field and self-gravity (called ISM-IT) on one hand and a simple isothermal simulation (called IT) on the other. Given the strong masking effect of thermal broadening discussed in the previous section, in this section we discuss only NTB cases so that the velocity structure is fully accessible to the SCF. However, note that this cannot be done in the case of real observational data.

Figures $5 a$ and $5 b$ show the velocity channels for cases ISM-IT and IT, respectively. The veil-like structure described for the ISM run is also present in the IT and ISM-
IT runs, although in the latter the veils are more roundish, somewhat resembling soap bubbles or peanut shells. This is most likely a reflection of the intermediate-scale forcing applied to the IT run (in scales of about $\frac{1}{4}$ of the box), in contrast with the small-scale forcing acting on the ISM and ISM-IT runs due to the stellar sources. The presence of the magnetic field in the ISM and ISM-IT runs is probably also a factor contributing to the more elongated structures present there. ${ }^{5}$

Another evident distinction between the runs is that very large-scale structures (of roughly half of the box size, running diagonally from one side of the box to the other), which are present in the ISM run (see, e.g., velocity channel at $\sim 4 \mathrm{~km} \mathrm{~s}^{-1}$ in Fig. $2 b$ ), are absent from cases IT and ISM-IT. This is because of the smaller effective Jeans length in the

\footnotetext{
${ }^{5}$ Note that in the ISM simulations, $\beta$, the magnetic to thermal pressure ratio, is less meaningful than in isothermal simulations since neither the magnetic or thermal pressure is constant, and, in fact, $\beta$ is a local quantity spanning a factor of at least 3 orders of magnitude.
} 


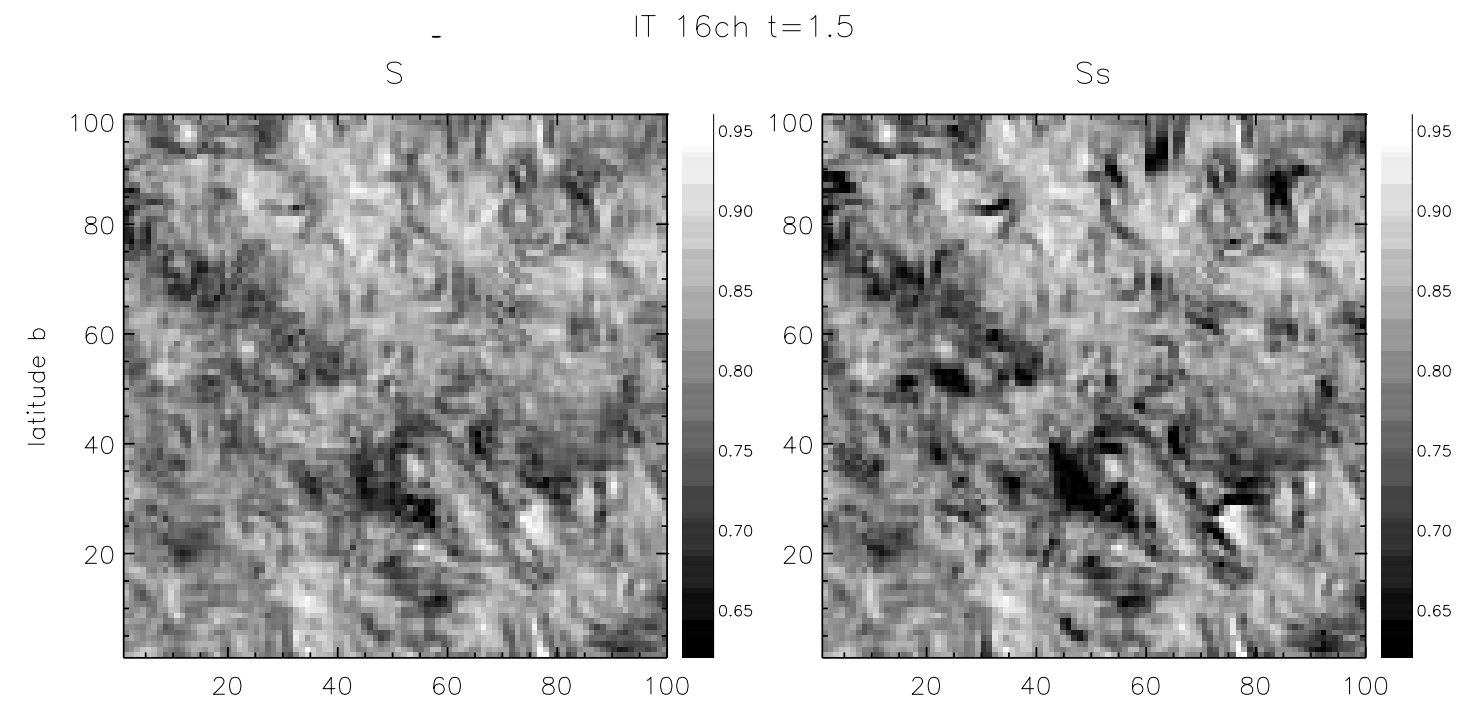

SI

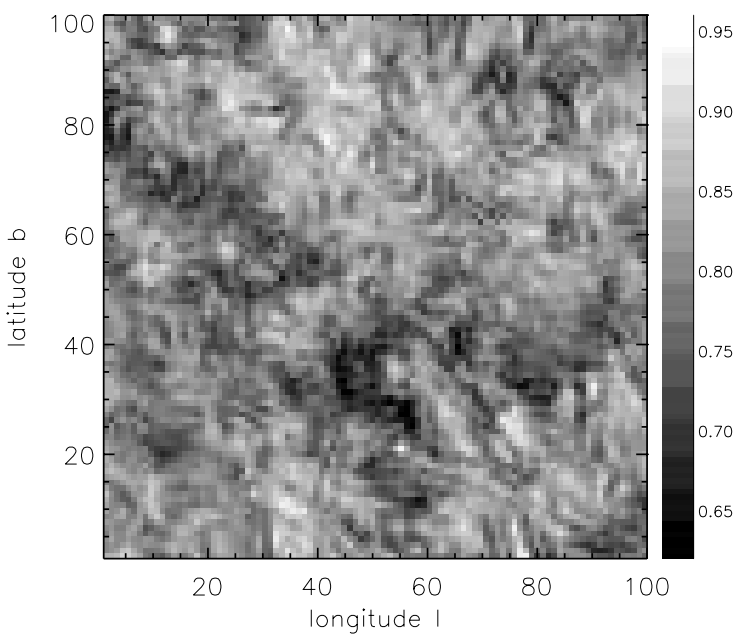

SO

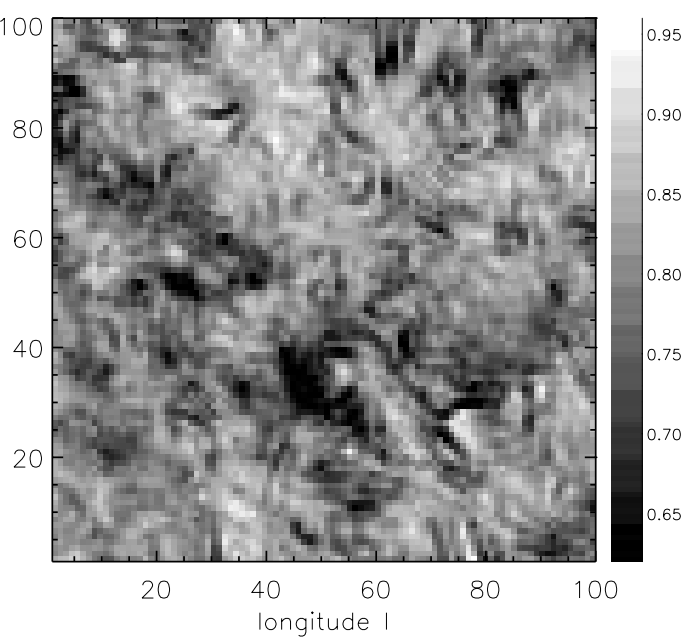

FIG. $6 b$

ISM run (§ 2.1). Thus, such large-scale structures in the ISM run have a gravitational origin but have no counterpart in the ISM-IT and IT runs.

The SCF maps of the ISM-IT and IT runs (Figs. $6 a$ and $6 b$ ) continue to show the "wormy" appearance described for the ISM run, although the "worms" form rounder bundles in the IT run because of the rounder shapes of the structures seen in its channel maps. The SCF histograms for all three runs (Figs. 4b, 7a, and $7 b$ ) have comparable means and variances. However, the histograms for runs in which star formation is active (ISM and ISM-IT) seem to have more extended low-correlation tails and, therefore, more negative values of the skewness. This appears to be a consequence of the greater abundance of shells and shocks in cases with active star formation and, thus, suggests that the histograms of the SCF are capable of " sensing" the presence of these structures, representing them by large negative skewness values. Unfortunately, the correspondence is not unique: the phenomena that can cause large low-SCF tails are not limited to shocks and shells. Any sharp changes in the gas structure, either in density or in LOS velocity or even in the PPV cubes (e.g., the caustics), can cause them. Thus, the method cannot be used to unambiguously identify shocks and shells. Instead, the existence of low-SCF tails in the histograms should be taken only as suggestive of their possible presence, which should then be verified by other means.

\subsection{SCF for the North Polar Loop}

We now apply the SCF to the H I maps of the NCP loop, a region possibly formed by a nearby supernova explosion (see, e.g., Pound \& Goodman 1997). In Figure $8 a$ we show the channel maps for this region, which contain an arcshaped structure appearing at positive velocities and a clear large-scale velocity gradient: at large negative velocities most of the emission is concentrated at high longitude and low latitude, while at large positive velocities most of the emission comes from the arc and is concentrated in the upper half of the region.

As we mentioned before ( $\S 2.2)$, the reasons for choosing this region are that it is a high-latitude region, avoiding the contribution to the emission from physically disconnected regions far away in the LOS, and that the determination of 

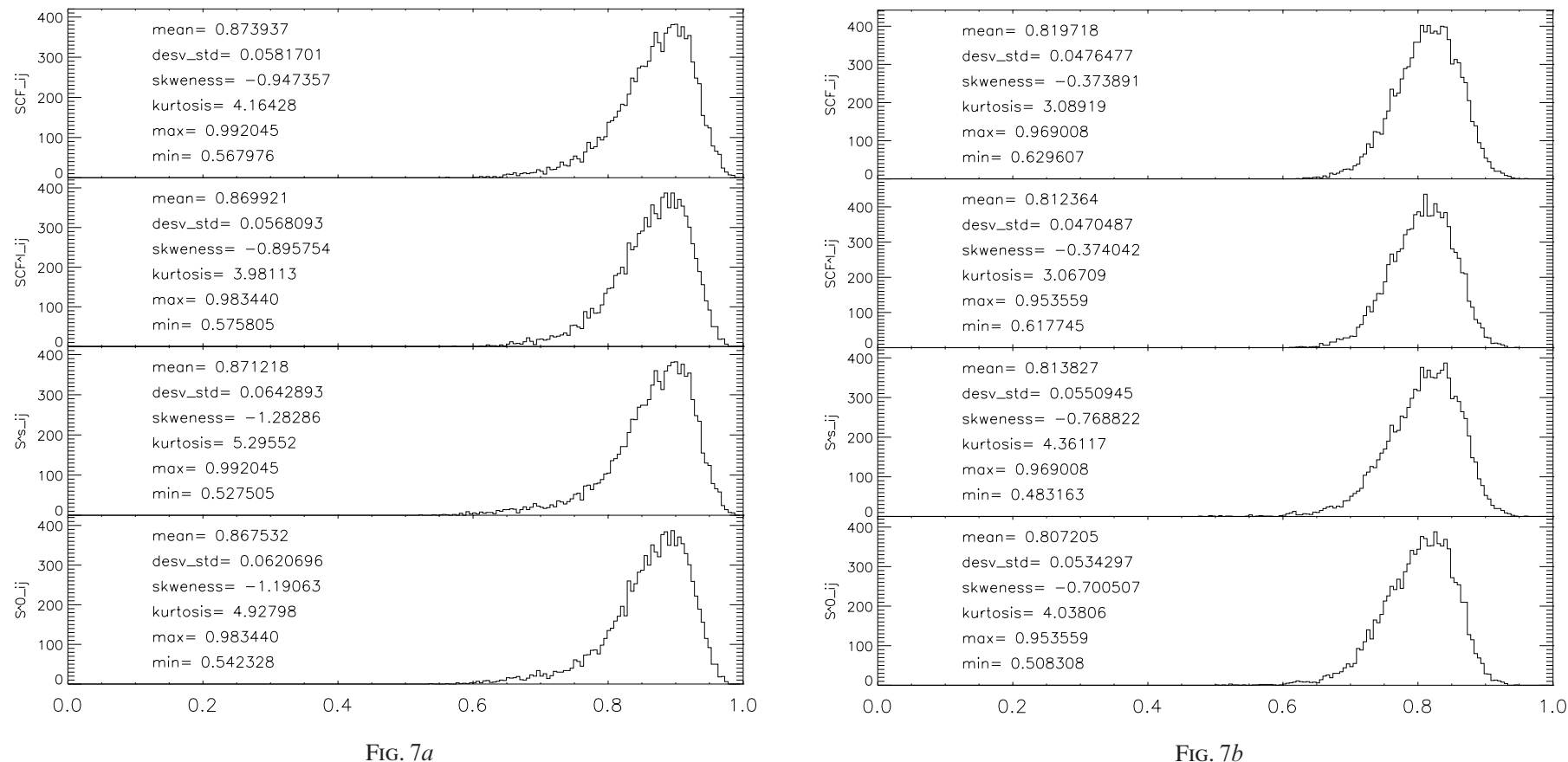

Fig. 7.- SCF histograms for Fig. 5. Although both runs have similar mean and variance, the run with star formation $(a)$ exhibits more extended lowcorrelation tails, suggesting that the SCF is able to pick up multiple shocks and shells.

the distance to some molecular clouds allows us to assume that the size of the region is about $160 \mathrm{pc}$, comparable to the size of the simulations ( $300 \mathrm{pc}$ ).

Figure $8 b$ then shows the SCF maps for this region. Several points are apparent. First, the "wormy" structure seen in all the simulations is also seen in the NCP loop, particularly in the arc region, indicating the presence of shocks or caustics in the field ( $\S \S 4.1 .1$ and 5). ${ }^{6}$ In this case, it is natural to assume that shocks are the primary cause of the "worms" given that the NCP loop is an expanding shell. Second, we see that the SCF maps only contain structure around the arc, and not near the bottom, where rather featureless emission is seen in the channel maps, indicating that the local form of the SCF used in this paper eliminates roughly uniform regions and highlights regions of strong variability. Another consequence of this behavior is that the local form of the SCF eliminates large-scale velocity gradients, as indicated by the fact that no sign of the gradient seen in the channel map set is apparent in the SCF maps. Although this is in a sense obvious, it makes the local SCF maps a powerful tool for studying the small-scale intensity and velocity structure. In particular, the SCF should make a good shell-identification tool in the sense that we can quantify, using the skewness of the histograms, the relative importance of sharp edges (shells) of gas.

Also worth noting is the fact that the map of $\mathrm{SCF}^{l}$ contains significantly lower values of the correlation compared to the map of $\mathrm{SCF}^{s}$, indicating that most of the variability is due to the intensity rather than to the velocity structure. This is also seen in the SCF histogram for $\mathrm{SCF}^{l}$ (Fig. 8c),

\footnotetext{
${ }^{6}$ The "worms" in the observations' SCF maps appear larger, at first sight, than those in the simulations' SCF maps. However, this is only due to the larger pixel size of the NCP loop maps. The filaments are in all cases only a few pixels wide.
}

which shows a more extended low-correlation tail and, consequently, a larger negative skewness than the $\mathrm{SCF}^{s}$ histogram. However, these features may instead be an indication that thermal broadening is not negligible for this region, thus partially "blinding" the SCF to the velocity structure. If this is so, then the thermal broadening should be of $3-5 \mathrm{~km} \mathrm{~s}^{-1}$, since the total width of the $\mathrm{H}_{\mathrm{I}}$ line profiles for this region is about $8-10 \mathrm{~km} \mathrm{~s}^{-1}$, such that the total width comes from the turbulent structure, but the thermal broadening does not allow such turbulent velocity structure to be seen in detail. Indeed, it is noteworthy that the histograms of $\mathrm{SCF}^{l}$ for the simulations have larger negative skewness values than those of $\mathrm{SCF}^{s}$ in the $\mathrm{TB}$ cases, but the opposite is true for the NTB cases, supporting this view. This is also probably the reason why the featureless emission at the bottom of the channel maps does not contain "worms" associated with the small-scale velocity structure in the SCF maps.

\section{DISCUSSION}

\section{1. "Worms" as Shocks or Caustics in PPV Space}

The local form of the SCF as used in this paper is, by definition, sensitive only to small-scale features (either in intensity or in velocity). Specifically, structural features in the SCF maps correspond to steep gradients in the POS of the line profiles' shape and/or amplitude. In particular, shocks and the caustics discussed by W. Henney et al. (2002, in preparation) are especially well suited for detection by the local SCF.

For clarity, a brief summary of the results of W. Henney et al. (2002, in preparation) is in order here. Essentially, the process of performing a spectroscopic observation amounts to performing a mapping from the real, three-dimensional physical space onto PPV space (see also Lazarian \& Pogosyan 2000; Pichardo et al. 


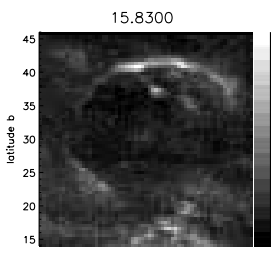

8.62000

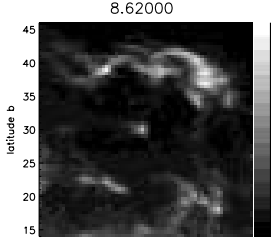

.41000

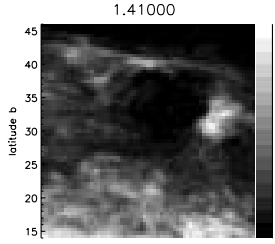

$-5.80000$

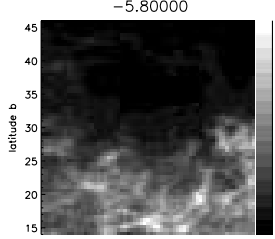

$-13.0100$

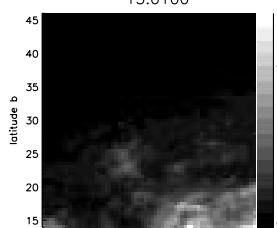

$-20.2200$

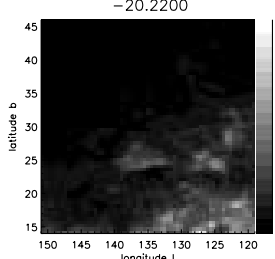

16.8600

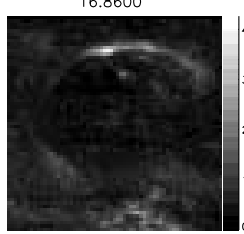

9.65000

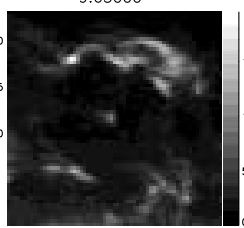

2.44000

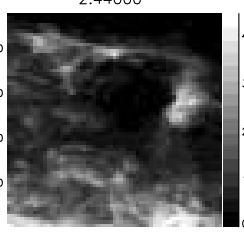

$-4.77000$

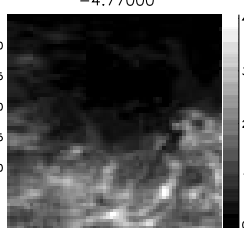

11.9800

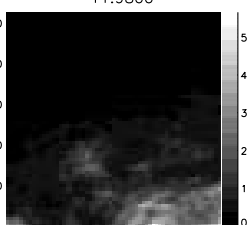

$-19.1900$

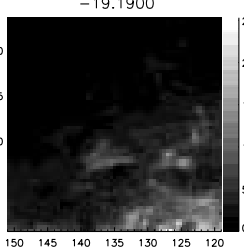

17.8900

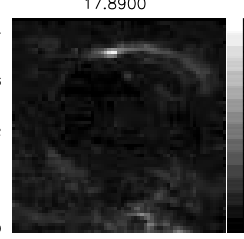

10.6800

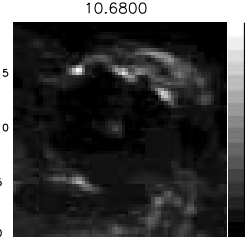

3.47000

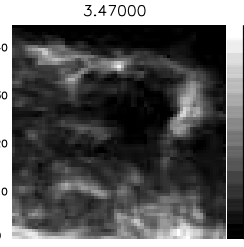

$-3.74000$

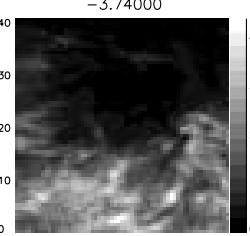

$-10.9500$

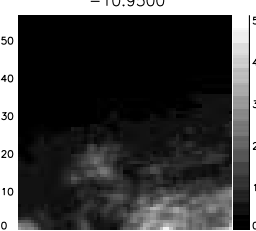

$-18.1600$

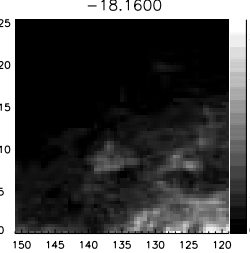

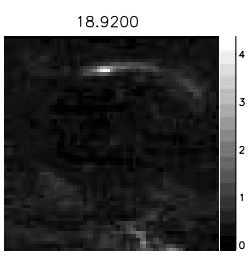

11.7100

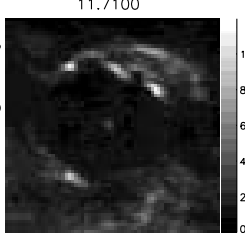

4.50000

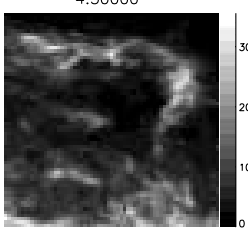

$-2.71000$

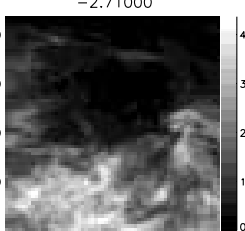

$-9.92000$

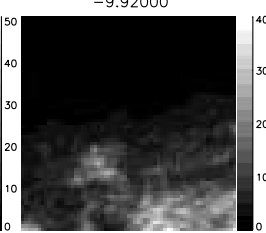

$-17.1300$

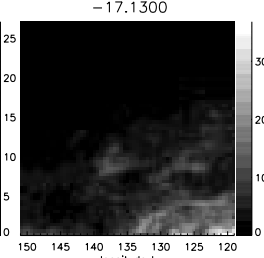

19.9500

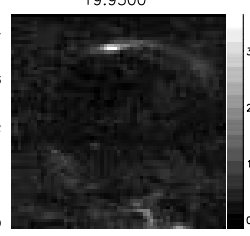

12.7400

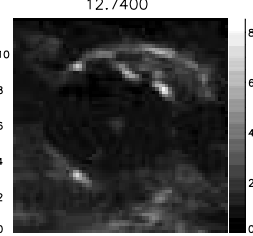

5.53000

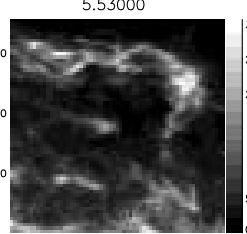

$-1.68000$

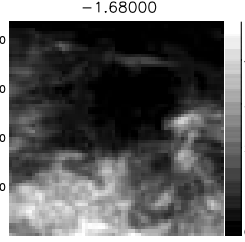

$-8.89000$

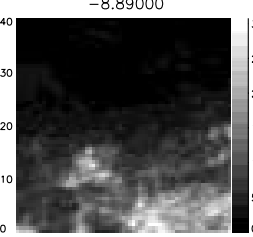

$-16.1000$

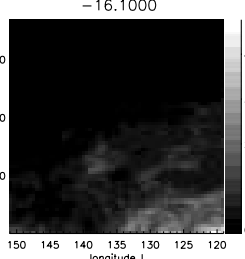

20.9800

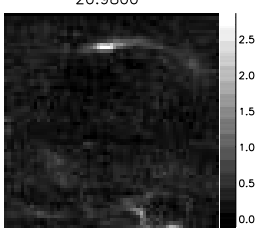

13.7700

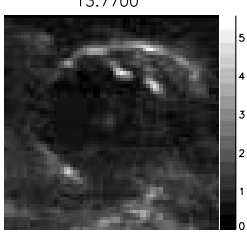

6.56000

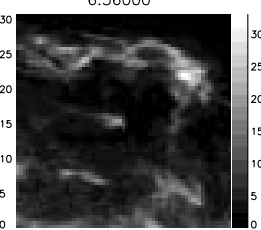

$-0.650000$

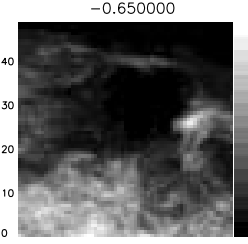

$-7.86000$

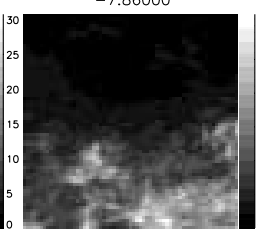

$-15.0700$

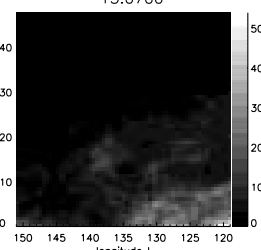

14.8000

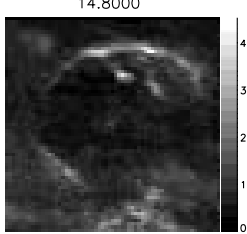

7.59000

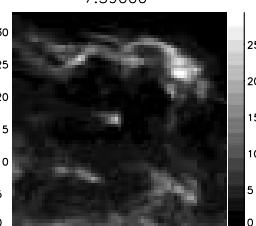

0.379999

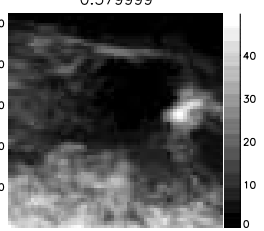

$-6.83000$

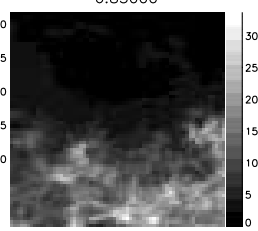

$-14.0400$

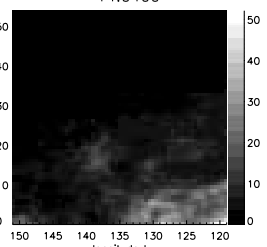

FIG. $8 a$

FIG. 8.- NCP loop (a) channel maps, (b) SCF maps, and (c) SCF histograms. As in the simulations, a "wormy" structure is seen in the maps, and low-SCF sites denote the places where shell-like structure is seen.

2000). As is well known, "caustics" form in PPV space at places where the mapping is not single-valued, i.e., where finite-length LOS segments contribute to infinitesimal velocity intervals, causing a diverging intensity at those sites in the PPV cube (a phenomenon known as "velocity crowding"). With finite-width velocity channels, the intensity does not diverge but still reaches large values at the caustics. The latter are surfaces in the PPV cube and, in general, intersect the velocity channels (constant-velocity planes in the PPV cube) at finite angles along lines which show up as elongated, wormlike features in the channel maps. This structure is spurious in the sense that it does not correspond to any actual density features in the original physical space. In the case of sharply defined velocity channels, the spurious structure reaches arbitrarily small scales as the velocity resolution is increased. Upon thermal broadening, the minimum scale present is explicitly calculated by W. Henney et al. (2002, in preparation).

This spurious small-scale structure may, however, be picked out by the local SCF, showing up as the "worms" we have mentioned repeatedly. However, if the thermal broadening is very strong (i.e., the velocities are strongly subsonic), the caustics become thicker and less intense in PPV space, so that the "worms" become wider and fainter. Thus, as already mentioned in $\S 4.1 .2$, the thermal broadening present in real spectroscopic observations has the fortunate effect of counteracting the spurious small-scale structures generated by the synthetic observation process, although at the cost of blurring the velocity information.

The other possible type of small-scale feature that is naturally picked out by the local SCF is shocked shells. 


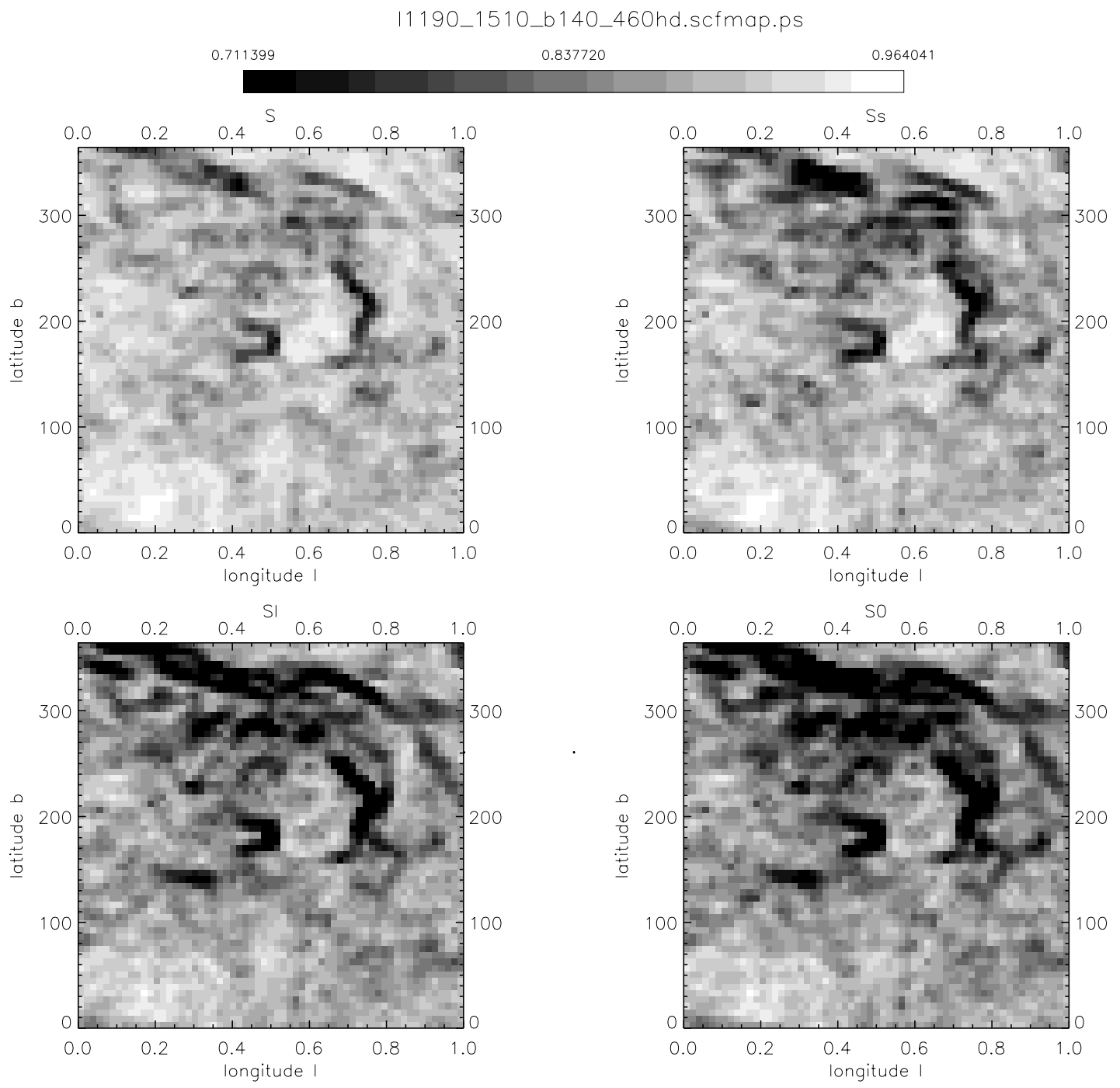

FIG. $8 b$

Contrary to the caustics, shocks are real features in three-dimensional space, and, since they imply supersonic velocity differences occurring over very small scales, it is possible that they "survive" thermal broadening. We conclude that when thermal broadening is not negligible "worms" in SCF maps are most likely to correspond to real velocity features-shocks. However, for extremely supersonic motions where thermal broadening is negligible, it is possible that the SCF may not be able to distinguish between them and the spurious caustics. Further work is necessary to quantify and distinguish caustics and shocks through the SCF.

\section{2. "Diagnosing" the Simulations through the $S C F$}

The SCF is intended to provide a quantitative means of comparing numerical simulations and observations, which should then allow workers to adjust their simulations to make them match the observational data as closely as possible. In this section we show an example of how this can be done.

Comparing both the NTB and the TB 16-channel SCF histograms of the ISM simulation (Figs. $4 a$ and $4 b$ ) with those of the NCP loop (Fig. 8c), we note that neither set of the simulation histograms is a very good match for those of the NCP loop and, instead, seem to bracket the latter. The NTB histograms are too broad and have smaller means than the NCP loop ones, while the opposite is true of the TB histograms. In particular, the narrowness of the TB histograms suggests that the thermal width dominates the line spectra of the simulation, causing them to be very similar, all being very close to Gaussians. Indeed, as seen in Figure 1, the TB profiles have lost essentially all the velocity information and are strongly dominated by the thermal component. Instead, the 16-channel NTB profiles are very spiky and do not look like realistic profiles.

As mentioned in $\S 4.1 .2$, we attribute this problem to the fact that even though the cold gas contains supersonic motions these motions are not supersonic compared to the thermal speed of the warm gas, and thus the velocity information of the cold gas is swamped in the thermal width of the warm gas. This is a real effect, and we expect it to affect the NCP loop data. However, it moreover occurs that the latter include the contribution from gas moving at significantly larger velocities, most likely because of large shell expansion velocities (see Pound \& Goodman 1997). Instead, 
11190_1510_b140_460hd.scf

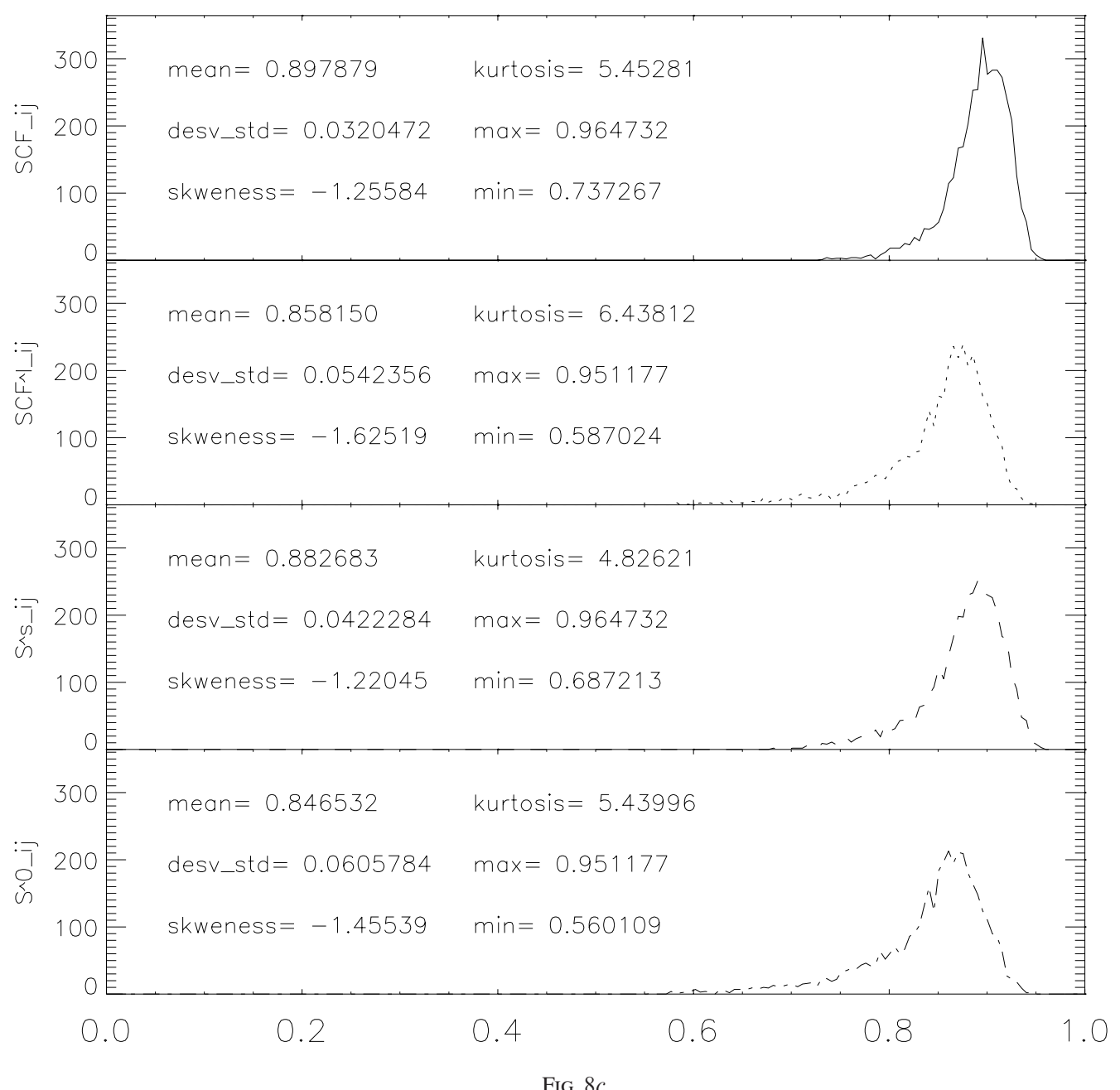

FIG. $8 c$

the simulation only contains motions in the interval $-6 \mathrm{~km} \mathrm{~s}^{-1} \lesssim v \lesssim 6 \mathrm{~km} \mathrm{~s}^{-1}$ since it includes only $\mathrm{H}$ II regionlike expanding shells but not supernova remnant-like ones, which would have much larger velocities. Thus, in the $\mathrm{H}$ I data there exists quite a large velocity range that "escapes" the thermal smoothing. This suggests that, in order to make the simulated line profiles more realistic, we should "expand" the LOS-velocity axis by some factor until the "right" ratio of nonthermal velocity range to thermal width is obtained. The expansion factor can be determined by matching the SCF histograms of the simulation with the thermal broadening and velocity expansion to those of the $\mathrm{H}$ I data. We find that expanding the velocity axis by a factor of 6 gives the best match, as shown in Figures $9 a$ and $9 b$, which present, respectively, velocity-expanded simulation data and some selected line profiles and the SCF histograms for the TB. Note also that, since the line profiles of Figure $9 a$ appear significantly more realistic, this figure also shows the extent to which the velocity structure is hidden by the thermal broadening, by comparing the solid lines (TB spectra) to the dotted lines (actual velocity histograms along the LOS).

Note that the matching can still be improved since other differences between the simulation and the NCP loop data still remain at the next level of refinement: first, although we have matched the mean and standard deviation of the SCF histograms, the skewness and kurtosis values of the simulation data are considerably smaller than those of the NCP loop data. This is a reflection of the fact that in the NCP loop channel SCF maps there exist regions of very low values of the SCF corresponding to the arc, which is a strong and large feature, while no such prominent structures are present in the simulation data. Second, the histograms of $\mathrm{SCF}^{l}$ have larger (negative) skewness values than the histograms of $\mathrm{SCF}^{s}$, while the opposite is true for the simulation histograms. As mentioned in $\S 4.3$, we have interpreted this as a consequence of the fact that in the NCP loop SCF maps the variability has a larger contribution from intensity variations, while in the simulation data there seems to be a larger contribution from velocity variation along an LOS. Thus, it seems that better matches could be obtained by comparing the simulation data to a region of sky without such a prominent feature, although for brevity we do not pursue this further here since we believe that the potential of the SCF to guide the simulations to match the observational data has been sufficiently exemplified.

Certainly, the velocity expansion we have done here is not a self-consistent fix to the simulation since the correct thing 

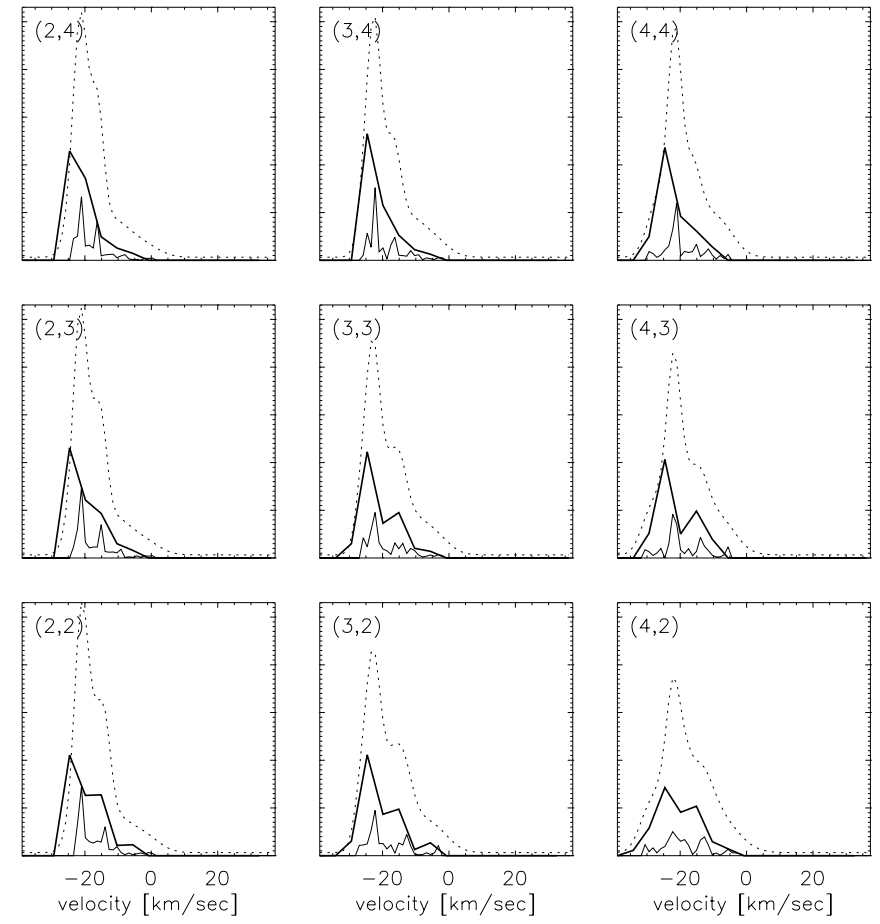

FIG. $9 a$
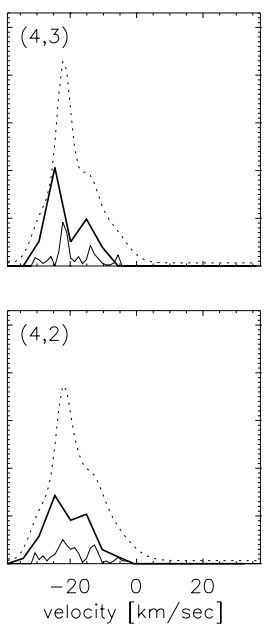

ISM100 TB. vz expanded 6 times

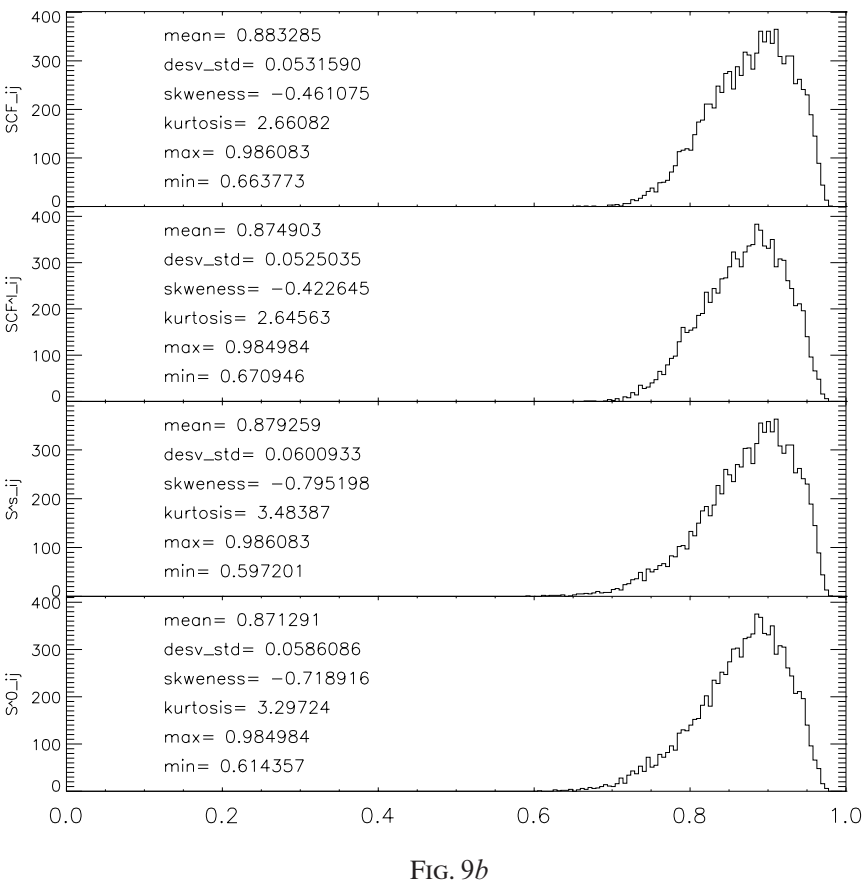

FIG. 9.- (a) Line profiles of the ISM run at the same positions as in Fig. 1 but with the velocity axis multiplied by a factor of 6 . The TB line profiles now appear more realistic than either the NTB or TB profiles of Fig. 1. (b) The SCF histograms are much closer in mean and standard deviation to those of the NCP loop data than either the NTB or the TB histograms shown in Figs. $4 a$ and $4 b$.

to do would be to rescale the whole simulation to Galactic scales and to correctly take into account the disk rotation and stratification, as well as more powerful sources of energy (supernovae) capable of accelerating the gas to higher velocities. The conclusion is that our simulations represent a much less turbulent medium than the actual ISM as implied by their smaller velocity dispersion compared to the velocity dispersion of the $\mathrm{H}$ I gas. This exercise has shown the potential of the SCF to pick out differences between the $\mathrm{H}$ I and simulation PPV data and to point toward the physical ingredients that are lacking in the simulations for them to be better models of a given type of observation.

\section{SUMMARY AND CONCLUSIONS}

In this paper we have presented further discussion on the spectral correlation function (SCF), in addition to that given by RGWW and PRG, and then applied it to data from numerical simulations of the ISM and from H I data (Hartmann \& Burton 1997) of the north celestial pole loop. The application to the numerical data, for which all the information on the distribution of the physical variables is known, allows us to understand the response of the various SCF measurements used here on the velocity structure in the simulations. This understanding was then used to interpret the results of applying the SCF to the NCP loop data and to suggest modifications to the parameters of the ISM simulation to better match the observational data.

In this paper, as in RGWW, the SCF has been used in a "local" form in which the SCF between one position in a map and its nearest neighbors is calculated and assigned as an "intensity" to that position, thus producing maps of the SCF. Thus, structural features in the SCF maps correspond to sharp gradients in the line profiles' shape and/or amplitude on the plane of the sky. Also, histograms of the SCF values in the maps have been studied. This procedure has been applied to all four "modes" of the $\mathrm{SCF}, \mathrm{SCF}^{0}, \mathrm{SCF}^{s}$, $\mathrm{SCF}^{l}$, and $\mathrm{SCF}^{l, s}$. The behavior of the $\mathrm{SCF}$ with separation, similar to the more familiar case of regular correlation functions in hydrodynamics (see, e.g., Lesieur 1990), is discussed by PRG.

We first reviewed basic definitions concerning the SCF, in particular the four modes that allow the SCF to focus on spectral similarities in either total intensity, velocity structure, none, or both. We then considered the effect of noise and showed that in the case of $\mathrm{H} \mathrm{I}$ (and certainly in the case of numerical simulations) it is not an issue, allowing us to neglect its effects in the subsequent discussion.

In the absence of thermal broadening, the SCF maps of all simulations exhibited a strikingly "wormy" structure, with an interesting kind of self-similarity in which the smallest scale "worms" bundle up to form larger-scale ones. The "worms" may be associated with caustics in the positionposition-velocity (PPV) data cube (loci of high intensity due to velocity crowding) discussed by W. Henney et al. (2002, in preparation). Such caustics are not a real feature of the physical fields but are generated by the spectroscopic observation process, and they are certainly present in the PPV cube, strongly influencing the structure seen in the channel maps. Their presence causes the SCF histograms to broaden and to have lower mean values and larger negative skewness values.

Application of the SCF to a multitemperature, selfgravitating MHD simulation of the ISM at intermediate scales (3-300 pc) showed that thermal broadening may dominate the spectral lines when the warm gas is primarily 
subsonic, even if cold, locally supersonic regions exist along the LOS (assuming optically thin lines) since these regions are still sub- or trans-sonic with respect to the warmer gas. This caused the small-scale structures (which also typically consists of smaller velocity amplitudes) to disappear from the TB SCF maps compared to the NTB ones and caused the TB SCF histograms to be much narrower and with higher mean values than their NTB counterparts. This implies that in the case of the multitemperature $\mathrm{H}$ I data much of the small-scale velocity structure associated with the colder gas may be unobservable in emission and is possibly only accessible through self-absorption features in the line profiles.

We then applied the SCF to two isothermal runs, one including stellar energy injection, self-gravity, and the magnetic field (ISM-IT), which may be representative of molecular gas, and one without all these ingredients and forced at large scales (IT). We did not consider thermal broadening for these runs because nearly isothermal (molecular) gas in the ISM is generally very supersonic, at least above scales of $\sim 0.1$ pc. The channel maps for these runs were seen to be markedly different from those for the ISM run, lacking the largest scale structures formed by large-scale gravitational instability in the latter run (the ISM-IT run has a Jeans length larger than the simulation size and the IT run has no gravity at all). However, SCF maps of the isothermal runs were not found to be so different because the "local" SCF used in this paper focuses on small-scale structures.

The SCF histograms of simulations ISM and ISM-IT, which have active star-forming regimes and thus contain stronger, more numerous shocks, tend to have more extended tails at low values of the SCF, and thus large negative skewness values. This appears to be due to the strong spectral and intensity variations caused by the shocks, suggesting that large negative skewness values in the SCF histograms are indicative of the presence of shocks and/or shells in the physical fields. Unfortunately, other structures in the gas, or even artifacts in the PPV cube such as the caustics, can also produce such low-SCF tails in the histograms, and thus the indication cannot be completely unambiguous.

Then, SCF maps and histograms of the NCP loop region were analyzed. The "wormy" structure is also seen in the maps for this case, indicating that the caustics are a real concern. Moreover, the histograms also exhibit large negative skewness values because of the prominence of the shell-like structure in this region, in agreement with the suggestion from the simulation data.

We then proceeded to show how the SCF can be used to "fine-tune" the simulations to better match the observations. Indeed, the means and standard deviations of the NTB and TB simulation histograms are seen to bracket the corresponding values for the NCP loop histograms, and in fact the TB line profiles are seen to be completely thermally dominated, with virtually no velocity information left. By attributing this effect to the fact that the ISM simulation contains a rather limited velocity contrast, we were able to obtain a much better match to the NCP loop SCF histograms and much more realistic line profiles by "expanding" the velocity range by a factor of 6 , indicating that the simulation requires stronger sources of energy to generate larger velocity dispersion. In other words, we found that the simulations presented here are less energetic than the gas in the NCP region.

We conclude that the local mode of the SCF used here has the ability to quantify the presence of specific velocity and structural features in observational data such as shocks and shells, as well as to allow a quantitative comparison between observational and simulation data.

We are pleased to acknowledge C. Heiles for enlightening and enjoyable discussions. This work was supported in part by NASA Astrophysical Theory Program grant NAG 510103 and CONACYT grants 88046-EUA to J. B.-P. and 27752-E to E. V.-S.
Avila-Reese, V., \& Vázquez-Semadeni, E. 2001, ApJ, 553, 645

Ballesteros-Paredes, J., Vázquez-Semadeni, E., \& Scalo, J. 1999, ApJ, 515, 286

Bensch, F., Stutzki, J., \& Osenkopf, V. 2001, A\&A, 366, 636

Brunt, C. M., \& Heyer, M. H. 2001, ApJ, 566, 276

Delgarno, A., McCray, R. A. 1972, ARA\&A, 10, 375

de Avillez, M. A. 2000, MNRAS, 315, 479

Dickman, R. L. 1985, in Protostars and Planets II, ed. D. C. Black \& M. S. Mathews (Tucson: Univ. Arizona Press), 150

Falgarone, E., Lis, D. C., Phillips, T. G., Pouquet, A., Porter, D. H., \& Woodward, P. R. 1994, ApJ, 436, 728

Franco, J., \& Carramiñana, A. 1999, Proc. 2nd Guillermo Haro Conf., Interstellar Turbulence (Cambridge: Cambridge Univ. Press)

Gazol, A., Vázquez-Semadeni, E., Sánchez-Salcedo, F. J., \& Scalo, J. 2001, ApJ, 557, L121

Gazol-Patiño, A., \& Passot, T. 1999, ApJ, 518, 748

Gibson, S. J., Taylor, A. R., Higgs, L. A., \& Dewdney, P. E. 2000, ApJ, 540,85

Hartmann, D., \& Burton, W. B. 1997, Atlas of Galactic Neutral Hydrogen (Cambridge: Cambridge Univ. Press)

Heiles, C. 2001, in ASP Conf. Ser. 231, Galactic Structure, Stars, and the Interstellar Medium, ed. C. E. Woodward, M. D. Bicay, \& J. M. Shull (San Francisco: ASP), 294

Heyer, M. H., \& Schloerb, F. P. 1997, ApJ, 475, 173

Houlahan, P., \& Scalo, J. 1992, ApJ, 393, 172

Kleiner, S. C., \& Dickman, R. L. 1984, ApJ, 286, 255

Lazarian, A., \& Pogosyan, D. 2000, ApJ, 537, 720

Lazarian, A., Pogosyan, D., Vázquez-Semadeni, E., \& Pichardo, B. 2001, ApJ, 555, 130

Lesieur, M. 1990, Turbulence in Fluids (Dordrecht: Kluwer)

Lis, D. C., Pety, J., Phillips, T. G., \& Falgarone, E. 1996, ApJ, 463, 623

Mac Low, M.-M., \& Ossenkopf, V. 2000, A\&A, 353, 339

\section{EFERENCES}

Miesch, M. S., \& Scalo, J. M. 1995, ApJ, 450, L27

Miesch, M. S., Scalo, J., \& Bally, J. 1999, ApJ, 524, 895

Padoan, P., \& Nordlund, A. 1999, ApJ, 526, 279

Padoan, P., Rosolowsky, E. W., \& Goodman, A. A. 2001, ApJ, 547, 862 (PRG)

Passot, T., Vázquez-Semadeni, E., \& Pouquet, A. 1995, ApJ, 455, 536 (PVP)

Penprase, B. E. 1993, ApJS, 88, 433

Pichardo, B., Vázquez-Semadeni, E., Gazol, A., Passot, T., \& BallesterosParedes, J. 2000, ApJ, 532, 353 .2000, ApJ, 532, 353

Pound, M. W., \& Goodman, A. A. 1997, ApJ, 482, 334

Raymond, J. C., Cox, D. P., \& Smith, B. W. 1976, ApJ, 204, 290

Rosen, A., \& Bregman, J. N. 1995, ApJ, 440, 634

Rosen, A., Bregman, J. N., \& Norman, M. L. 1993, ApJ, 413, 137

Rosolowsky, Goodman, A. A., Wilner, D., \& Williams, J. P. 1999, ApJ, 524, 887 (RGWW)

Scalo, J. 1987, in Proc. Symp. on Interstellar Processes; ed D. J. Hollenbach, \& H. A. Thronson, Jr. (Dordrecht: Reidel), 349

1990, in Proc. Workshop on Physical Processes in Fragmentation and Star Formation, ed. R Capuzzo-Dolcetta, C. Chiosi, \& A. Di Fazzio (Dordrecht: Kluwer), 151

Stutzki, J., Bensch, F., Heithausen, A., Ossenkopf, V., \& Zielinsky, M. 1998, A\&A, 336, 697

Vázquez-Semadeni, E., Ballesteros-Paredes, J., \& Rodriguez, L. F. 1997, ApJ, 474, 292

Vázquez-Semadeni, E., Gazol, A., \& Scalo, J. 2000a, ApJ, 540, 271

Vázquez-Semadeni, E., Ostriker, E. C., Passot, T., Gammie, C. F., \& Stone, J. M. 2000b, Protostars and Planets IV, ed. V. Mannings, A. P. Boss, S. S. Russell (Tucson: Univ. Arizona Press), 3

Vázquez-Semadeni, E., Passot, T., \& Pouquet, A. 1996, ApJ, 473, 881

Zuckerman, B., \& Evans, N. J. 1974, ApJ, 192, L149 